\title{
MST1-dependent vesicle trafficking regulates neutrophil transmigration through the vascular basement membrane
}

\author{
Angela R.M. Kurz, ${ }^{1}$ Monika Pruenster, ${ }^{1}$ Ina Rohwedder, ${ }^{1}$ Mahalakshmi Ramadass, ${ }^{2}$ Kerstin Schäfer, ${ }^{3}$ Ute Harrison, ${ }^{4}$ \\ Gabriel Gouveia, ${ }^{1,5}$ Claudia Nussbaum, ${ }^{1,6}$ Roland Immler, ${ }^{1}$ Johannes R. Wiessner, ${ }^{1}$ Andreas Margraf, ${ }^{1}$ Dae-Sik Lim, ${ }^{7}$ \\ Barbara Walzog, ${ }^{1}$ Steffen Dietzel, ${ }^{1}$ Markus Moser, ${ }^{8}$ Christoph Klein, ${ }^{6}$ Dietmar Vestweber, ${ }^{3}$ Rainer Haas, ${ }^{4}$ \\ Sergio D. Catz, ${ }^{2}$ and Markus Sperandio ${ }^{1}$
}

'Walter Brendel Centre of Experimental Medicine, Ludwig Maximilians University of Munich, Munich, Germany. ${ }^{2}$ Department of Molecular and Experimental Medicine, The Scripps Research Institute, La Jolla, California, USA. ${ }^{3}$ Department of Vascular Cell Biology, Max Planck Institute for Molecular Biomedicine, Münster, Germany. ${ }^{4}$ Max von Pettenkofer-Institute for Hygiene and Medical Microbiology, Ludwig Maximilians University of Munich, Munich, Germany. ${ }^{5}$ CAPES Foundation, Ministry of Education of Brazil, Brasilia, Brazil. 'Dr. Von Hauner Children's Hospital, Ludwig Maximilians University of Munich, Munich, Germany. ${ }^{7}$ Department of Biological Sciences, Biomedical Research Center, Korea Advanced Institute of Science and Technology, Daejon, Korea. ${ }^{8}$ Department of Molecular Medicine, Max Planck Institute of Biochemistry, Martinsried, Germany.

Neutrophils need to penetrate the perivascular basement membrane for successful extravasation into inflamed tissue, but this process is incompletely understood. Recent findings have associated mammalian sterile 20-like kinase 1 (MST1) loss of function with a human primary immunodeficiency disorder, suggesting that MST1 may be involved in immune cell migration. Here, we have shown that MST1 is a critical regulator of neutrophil extravasation during inflammation. Mst1-deficient $\left(M s 1^{-1-}\right)$ neutrophils were unable to migrate into inflamed murine cremaster muscle venules, instead persisting between the endothelium and the basement membrane. Mst $1^{1_{-}^{-}}$neutrophils also failed to extravasate from gastric submucosal vessels in a murine model of Helicobacter pylori infection. Mechanistically, we observed defective translocation of VLA-3, VLA-6, and neutrophil elastase from intracellular vesicles to the surface of $\mathrm{Mst1}^{-/-}$neutrophils, indicating that MST1 is required for this crucial step in neutrophil transmigration. Furthermore, we found that MST1 associates with the Rab27 effector protein synaptotagmin-like protein 1 (JFC1, encoded by Syt/1 in mice), but not Munc13-4, thereby regulating the trafficking of Rab27-positive vesicles to the cellular membrane. Together, these findings highlight a role for MST1 in vesicle trafficking and extravasation in neutrophils, providing an additional mechanistic explanation for the severe immune defect observed in patients with MST1 deficiency.

\section{Introduction}

Recruitment of neutrophils from the intravascular compartment to sites of inflammation is a fundamental process during the innate immune response. The process follows a well-defined cascade of events, including neutrophil capture and rolling, firm adhesion, crawling, extravasation, and migration into inflamed tissue (1). Although most of the recruitment steps have been thoroughly studied in the past (2), the precise mechanism of neutrophil transmigration across venular walls and penetration of the perivascular basement membrane (BM) remains elusive (3-6). Once neutrophils have migrated through the endothelial layer, they are primed for subsequent penetration of the endothelial BM (7-9) at sites that express low levels of laminin 511 (10-12) and potentially also low levels of collagen IV (13). So far, it is known that the surface expression of VLA-3 (integrin $\alpha_{3} \beta_{1}$, also known as CD49c/CD29) (14) and VLA-6 (integrin $\alpha_{6} \beta_{1}$, also known as CD49f/CD29) (15), 2 laminin-binding integrins on neutrophils, is required for migration

Conflict of interest: The authors have declared that no conflict of interest exists. Submitted: February 15, 2016; Accepted: August 25, 2016.

Reference information: / Clin Invest. 2016;126(11):4125-4139. doi:10.1172/JCI87043. into inflamed tissue. VLA-3 was shown to be necessary for neutrophil extravasation in TNF- $\alpha$ - and fMLP-induced transmigration in vivo $(14,16)$, while studies using blocking antibodies suggest a role for VLA- 6 in IL-1 $\beta$-dependent, but not TNF- $\alpha$-dependent, neutrophil extravasation (15). Migration through the BM was described to be further accompanied by the surface expression of the serine protease neutrophil elastase (NE) (17), although experiments using NE-deficient mice in vivo could not confirm a role in the extravasation process (18). Since VLA-3, VLA-6, and NE are stored in intracellular vesicles $(17,19)$, they need to be translocated to the cell surface during transmigration. Rab27 GTPases are involved in vesicle trafficking in neutrophils (20). Rab27a has emerged as a central regulator of many neutrophil functions through its ability to regulate vesicle exocytosis (21). So far, 2 effector molecules, namely synaptotagmin-like protein 1 (JFC1, encoded by Sytl1 in mice) and Munc13-4 (UNC13D), have been identified as interacting with Rab27a in neutrophils (22-25).

Recently, 3 independent groups identified a novel human primary immunodeficiency disorder, caused by autosomal recessive loss-of-function mutation in serine/threonine protein kinase 4 (STK4), which encodes the mammalian sterile 20-like kinase 1 
A

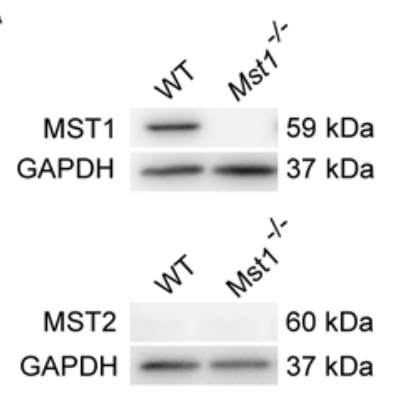

C

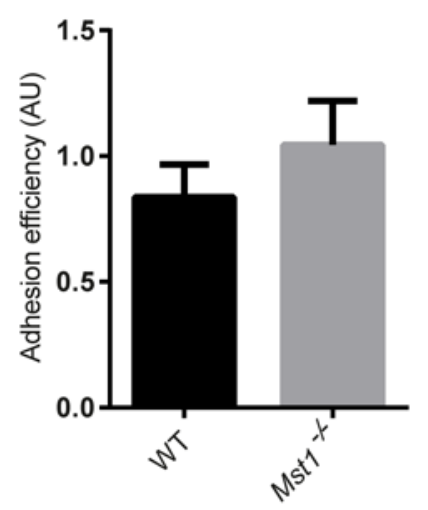

$\mathbf{F}$

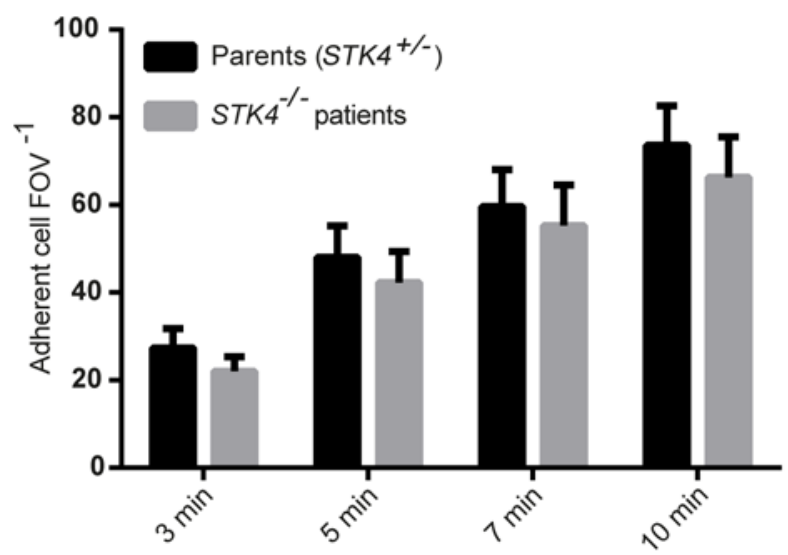

B

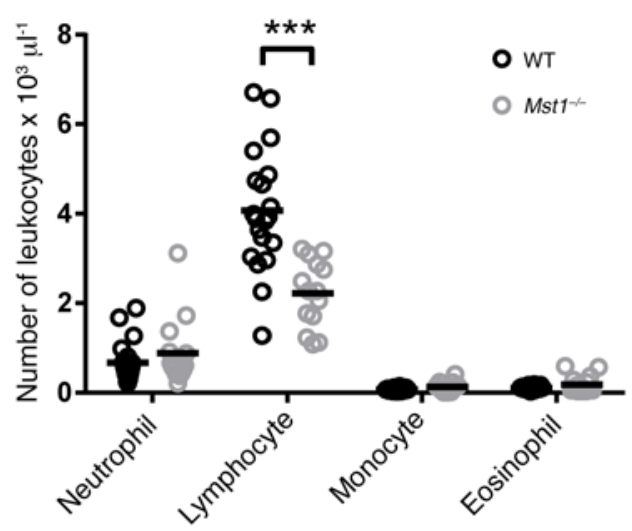

D

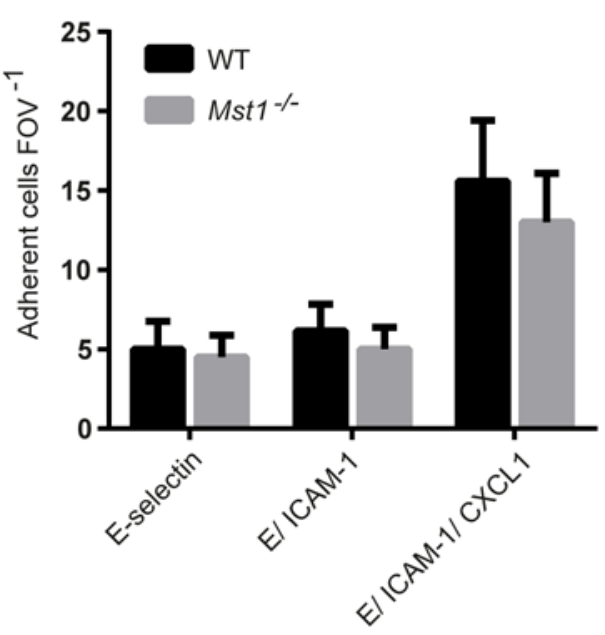

E
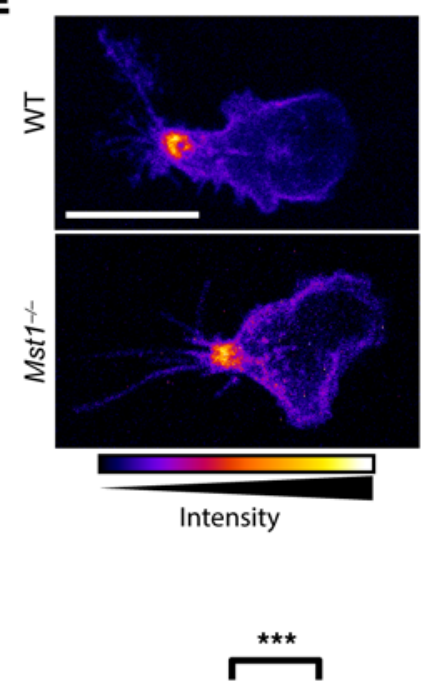

G

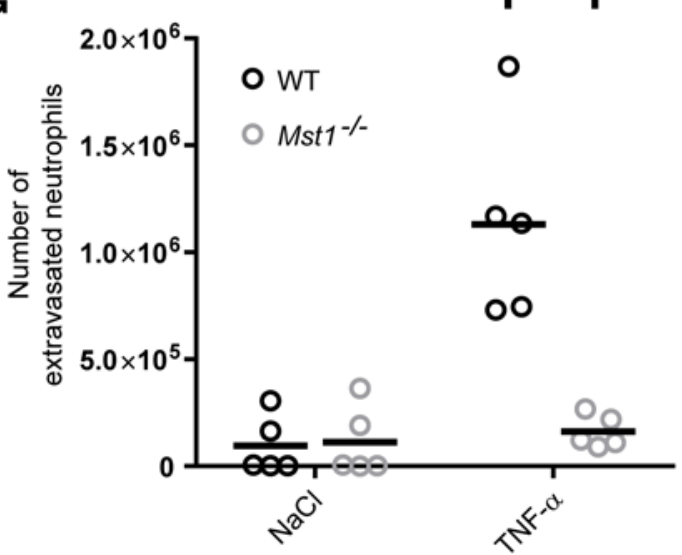

Figure 1. MST1 is dispensable for neutrophil adhesion in humans and mice, but critical for neutrophil extravasation into inflamed peritoneum. (A) MST1 and MST2 protein levels of WT and Mst1 $1^{-/-}$neutrophils. GAPDH served as loading control $(n=3)$. (B) Differential blood counts of WT ( $\left.n=20\right)$ and Mst1 ${ }^{-1-}$ mice $(n=14)$ (scatter plot with mean, ${ }^{* * *} P<0.001,2$-way ANOVA, Sidak's multiple comparisons test). (C) Neutrophil adhesion efficiency (arbitrary units) in cremaster muscle venules of WT and Mst1 $1^{-/-}$mice, 2 hours after intrascrotal injection of TNF- $\alpha(n=5$, mean \pm SEM, NS, unpaired $t$ test). (D) Number of adherent leukocytes per field of view (FOV) in microflow chambers coated with E-selectin, E-selectin/ICAM-1, or E-selectin/ICAM-1/CXCL1 ( $n=3$, mean \pm SEM, NS, 2-way ANOVA, Sidak's multiple comparisons test). (E) LFA-1 clustering of WT or Mst1-/- cells labeled with anti-LFA-1 antibody conjugated with Alexa 546 (fluorescence intensity scale). Whole blood was perfused through microflow chambers coated with E-selectin, ICAM-1, and CXCL1 (Supplemental Videos 1 and 2) ( $n=3$ mice, 1 representative picture is shown). Scale bar: $10 \mu \mathrm{m}$. (F) Number of adherent neutrophils per field of view from 2 patients with STK4 deficiency and their heterozygous parents was calculated over time in flow chambers coated with E-selectin/ICAM-1/CXCL8 ( $n=2$, chambers $\geq 2$, mean \pm SEM). (G) Total number of extravasated neutrophils in the peritoneal lavage 2 hours after i.p. injection of NaCl or TNF- $\alpha$ in WT and Mst1 $7^{-1-}$ mice ( $n=5$, scatter plot with mean, ${ }^{* *} P<0.001,2$-way ANOVA, Tukey's multiple comparisons test). 
(MST1), the mammalian homolog of the Drosophila melanogaster kinase Hippo. Children with STK4 deficiency display recurrent bacterial, viral, and fungal infections together with autoimmune disease. They also suffer from lymphopenia, which explains part of the clinical manifestations. However, their impaired response to acute bacterial infections also suggests a defect in myeloid cell function (26-28).

Studies using Mst1-deficient mice showed that the ubiquitously expressed serine/threonine kinase MST1 is involved in lymphocyte trafficking by controlling exocytosis and localization of the $\beta_{2}$ integrin lymphocyte function-associated antigen-1 (LFA-1, also known as CD11a/CD18 or integrin $\alpha_{\mathrm{L}} \beta_{2}$ ) to the plasma membrane. MST1 associates via RAPL with the cytoplasmic tail of the $\alpha_{L}$ subunit and leads to the translocation of LFA-1-containing vesicles in a DENND1C/Rab13-dependent manner (29). The absence of MST1 in lymphocytes leads to adhesion as well as migration defects, resulting in impaired lymphocyte trafficking and a decreased number of peripheral lymphocytes $(30,31)$. To investigate the role of MST1 for myeloid cell function, we evaluated neutrophil recruitment in $\mathrm{Mst1}^{-/-}$mice under in vivo and in vitro conditions. We show that MST1 is dispensable for LFA-1dependent neutrophil adhesion in mice and humans. In contrast, MST1 is critical for the translocation of vesicles containing VLA3, VLA-6, and NE to the neutrophil surface, by cooperating with the Rab27 effector protein JFC1. This translocation enables neutrophils to penetrate the $\mathrm{BM}$ and extravasate into the interstitial space. Hence, we identify MST1 deficiency as a neutrophil transmigration deficiency with a BM penetration defect due to the role of MST1 in vesicle trafficking.

\section{Results}

MST1 is dispensable for neutrophil adhesion but critical for extravasation. In the past, conflicting results on the expression of MST1 in mouse neutrophils were published (32). Therefore, we first confirmed the expression of MST1 in neutrophils from WT and the absence of MST1 in $\mathrm{Mst1}^{-/-}$neutrophils (Figure 1A). Notably, we did not detect any MST2 expression either in WT or in $\mathrm{Mst1}^{-/-}$neutrophils (Figure 1A and Supplemental Figure 1A; supplemental material available online with this article; doi:10.1172/ JCI87043DS1). Next, we determined differential cell counts of peripheral blood, and our results are in accordance with previously published data (33) showing decreased numbers of lymphocytes in $\mathrm{Mst1}^{-/-}$mice compared with WT mice (Figure 1B). In contrast, neutrophil counts were not significantly altered in $\mathrm{Mst1}^{-1-}$ mice compared with WT mice. Since lack of MST1 enhanced susceptibility to apoptosis in T cells $(31,34)$ and in neutrophils from STK4-deficient patients (26), we evaluated whether MST1 deficiency influences apoptosis of murine neutrophils. We found that neutrophils from WT and $\mathrm{Mst1}^{-/-}$mice showed no significant difference in the viability and apoptosis rate in vitro and in vivo (Supplemental Figure 1, B and C).

Previous studies had shown that MST1 is crucial for T cell adhesion dependent on the $\beta_{2}$ integrin LFA- 1 (30). To test whether LFA-1-dependent adhesion is also affected in neutrophils lacking MST1, we compared neutrophil adhesion efficiency in TNF- $\alpha-$ stimulated cremaster muscle venules of WT and $\mathrm{Mst1}^{-/-}$mice using intravital microscopy. Unexpectedly, we observed no dif- ference in neutrophil adhesion efficiency between WT and $M s t 1^{-/-}$ mice (Figure 1C). In addition, neutrophil rolling flux fraction and neutrophil rolling velocity, which is regulated by LFA-1, were not altered in $\mathrm{Mst1}^{-/}$mice compared with WT mice (Supplemental Figure 1, D and E). We then performed ex vivo flow chamber assays using glass capillaries coated with various combinations of adhesion molecules and found no difference in the number of adherent leukocytes (Figure 1D). Furthermore, $\mathrm{Mst1}^{-/-}$neutrophils displayed proper LFA-1 reorganization and accumulation at the uropod during postarrest modifications as observed in flow chambers using confocal microscopy (Figure 1E and Supplemental Videos 1 and 2). In addition, CXCL1-dependent LFA-1 activation in a soluble ICAM-1 binding assay was similar between neutrophils isolated from WT and $\mathrm{Mst1}^{-/-}$mice (Supplemental Figure 1, F and G). Neutrophil surface expression of LFA-1, Mac-1, CXCR2, CD44, PSGL1, and CD62L was similar in WT and $\mathrm{Mst1}^{-/-}$mice (Supplemental Figure $1 \mathrm{H})$.

Next, we investigated adhesion of neutrophils from 2 human patients with STK4 (also known as MST1) deficiency and their heterozygous parents (26). For this approach, we determined the number of adherent neutrophils per field of view in flow chambers coated with E-selectin/ICAM-1/CXCL8. The number of adherent neutrophils per field of view of $S T K 4^{-/-}$patients was similar to adhesion observed for chambers perfused with neutrophils from their heterozygous $\left(S T K 4^{+/-}\right)$parents (Figure $1 \mathrm{~F}$ ).

We then went on and asked whether MST1 deficiency could have any impact on neutrophil extravasation in a TNF- $\alpha$-induced peritonitis model. In WT mice, i.p. injection of TNF- $\alpha$ dramatically increased the number of neutrophils in the peritoneum compared with $\mathrm{NaCl}$ control injection. In contrast, neutrophils from $M s t 1^{-/-}$mice failed to transmigrate into the peritoneal cavity within 2 hours of TNF- $\alpha$ stimulation (Figure 1G).

Taken together, these in vitro and in vivo results demonstrate that, in contrast to T cells, MST1 is dispensable for LFA-1 activation and clustering in neutrophils. Hence, neutrophil adhesion is unaffected in $\mathrm{Mst1}^{-/-}$mice as well as in patients with STK4 deficiency. However, we could detect a massive decrease in the number of extravasated neutrophils in the peritoneal lavage of $\mathrm{Mst1}^{-/-}$ mice compared with WT mice in the TNF- $\alpha$-induced peritonitis model, suggesting a neutrophil recruitment defect downstream of intravascular adhesion.

Loss of MST1 in neutrophils results in decreased transmigration in vivo. Using multiphoton laser scanning microscopy, we next investigated neutrophil transmigration 180-240 minutes after intrascrotal injection of TNF- $\alpha$, together with a nonblocking antibody against PECAM-1 (35), into $L y z 2^{\text {GFP }}$ (36) and $M s t 1^{-/-} \times L y z 2^{G F P}$ mice, where neutrophils are endogenously labeled with EGFP. We observed an increasing number of neutrophils in $L y z 2^{G F P}$ mice crossing postcapillary venules and migrating into the inflamed tissue (Figure 2A, top panel, and Supplemental Video 3). In contrast, neutrophils from $\mathrm{Mst1}^{-/-} \times L y z 2^{\mathrm{GFP}}$ mice remained close to the abluminal part of the vessel wall (Figure 2A, bottom panel, and Supplemental Video 4). We then determined the duration of neutrophil transmigration into tissue and observed that transmigration of $L y z 2^{G F P}$ mice required $7.5 \pm 0.8$ minutes (median $\pm \mathrm{SEM}$ ), which is similar to the time reported by others (35). In contrast, most neutrophils of $\mathrm{Mst1}^{-/-} \times \mathrm{Lyz}^{\mathrm{GFP}}$ mice failed to transmigrate into tissue 
A

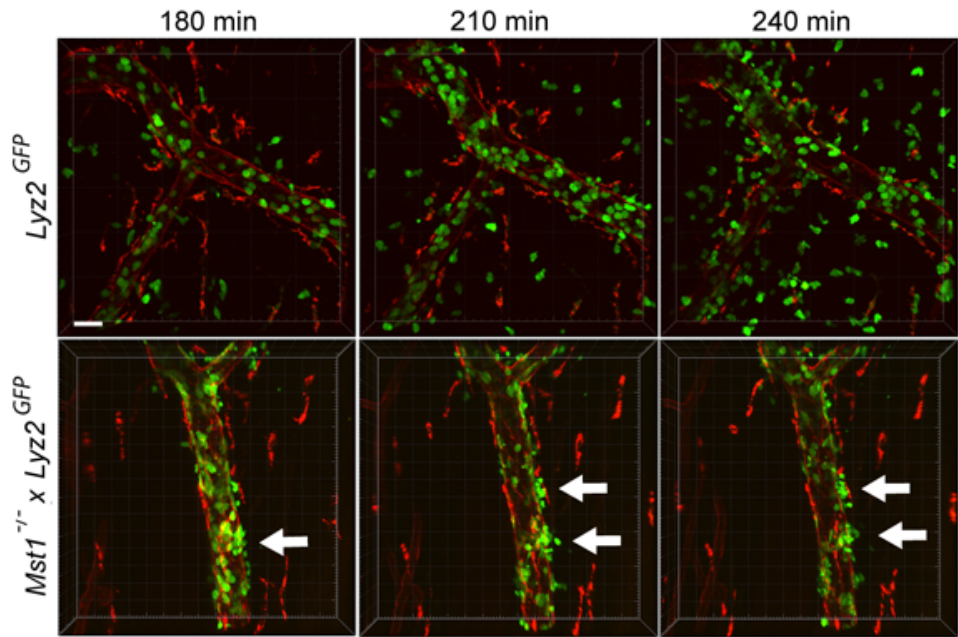

C
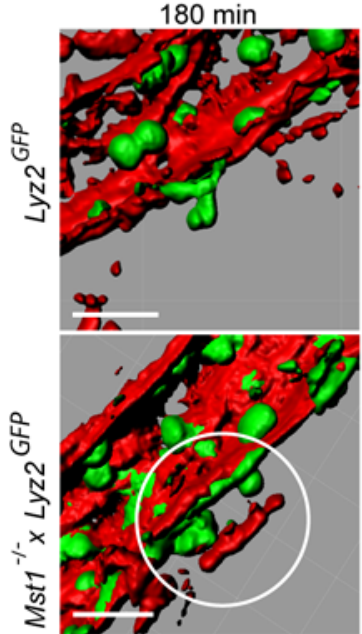

D

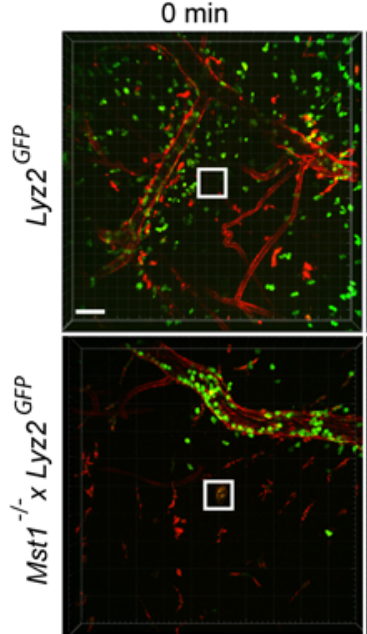

$210 \mathrm{~min}$
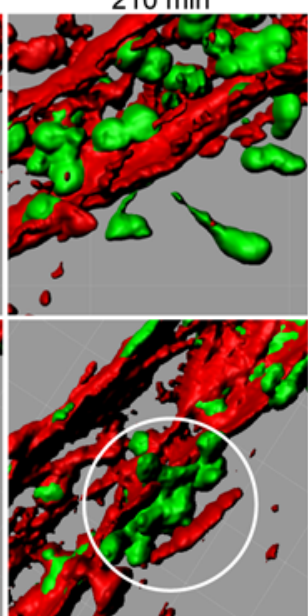

$240 \mathrm{~min}$
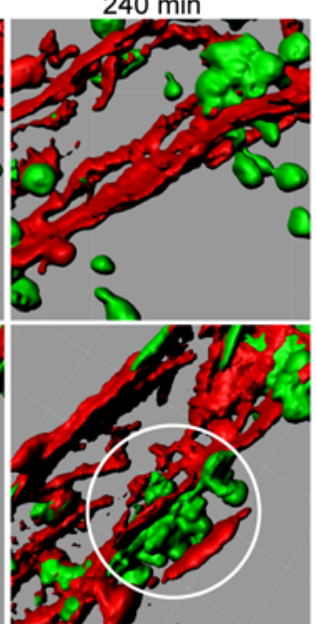

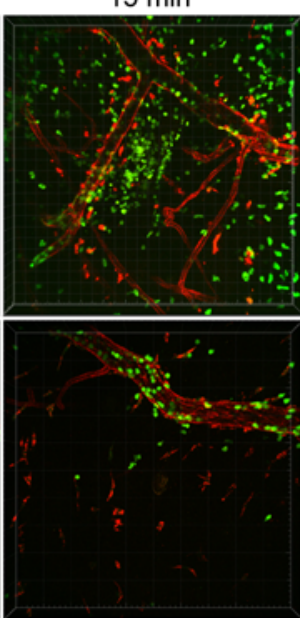

$30 \mathrm{~min}$

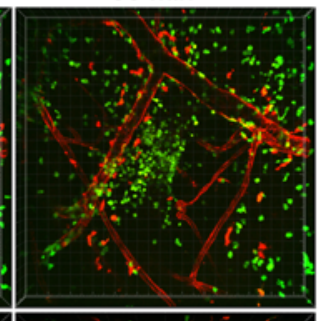

B

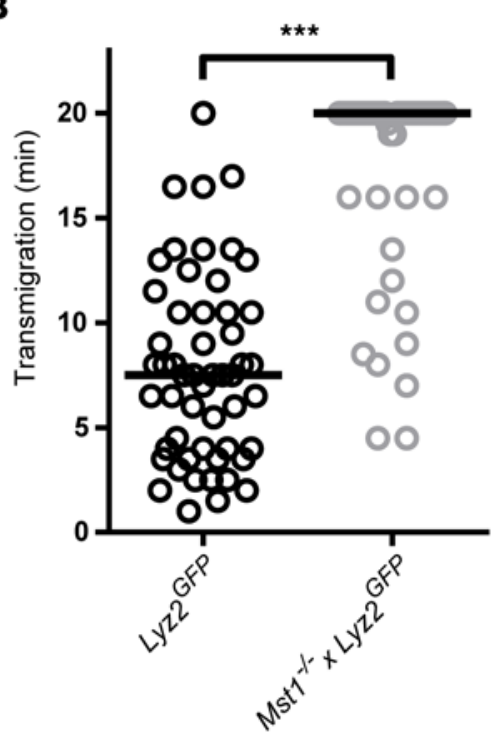

E

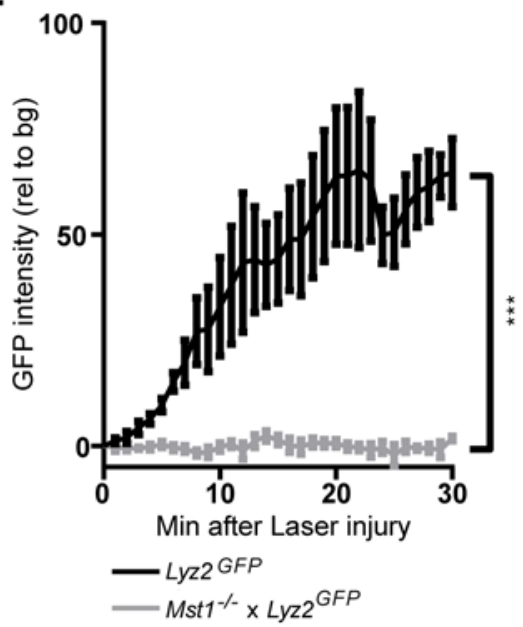

Figure 2. MstT ${ }^{-1-}$ neutrophils fail to transmigrate into inflamed tissue. Neutrophils (green) and microvasculature (red, anti-PECAM-1) of TNF- $\alpha$-stimulated mouse cremaster muscle. (A) Neutrophil transmigration in $L y z 2^{C F P}$ (top panels) and Lyz $2^{\mathrm{CFP}} \times \mathrm{Mst1}^{-1-}$ mice (bottom panels, white arrows) at 180 minutes, 210 minutes, and 240 minutes after intrascrotal injection of TNF- $\alpha$. Images were obtained using intravital multiphoton microscopy $(n=5)$. Scale bar: $30 \mu \mathrm{m}$. (B) Transmigration time of $\angle y z z^{C F P}$ and $L y z 2^{C F P} \times M s t 1^{-1-}$ neutrophils. Cutoff was set at 20 minutes $(n=5$ mice, scatter plot with median of $>50$ analyzed transmigration events per group, ${ }^{* *} P<0.001$, Mann-Whitney test). (C) 3D reconstruction of neutrophil (green) transmigration with subendothelial accumulation of $L y z 2^{C F P} \times M s t 1^{-1-}$ neutrophils (white circles) and complete extravasation of $L y z 2^{C F P}$ neutrophils. Scale bar: $20 \mu \mathrm{m}$. (D) Representative images of swarming neutrophils (green) and the microvasculature (PECAM-1, red) in the cremaster muscle of $L y z 2^{\mathrm{CFP}}$ and $L y z 2^{\mathrm{CFP}} \times M s t 1^{-1-}$ mice after induction of a laser injury (white squares). Scale bar: $50 \mu \mathrm{m}$. (E) Intensity profile of the GFP signal relative to background (rel to bg) in $L y z 2^{\text {CFP }}$ and $L y z 2^{\text {CFP }} \times$ $\mathrm{Mst1}^{-1-}$ mice at the site of laser injury $\left(n=5\right.$, mean $\pm \mathrm{SEM},{ }^{* *} P<0.001$ after 16 minutes, 2-way ANOVA, Sidak's multiple comparisons test). 
A
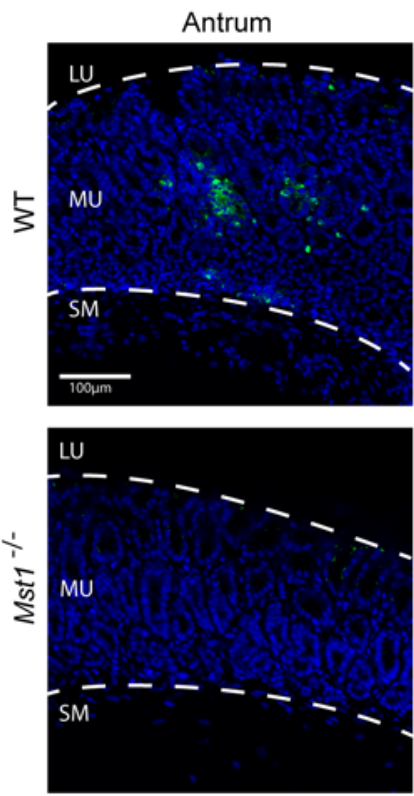

D
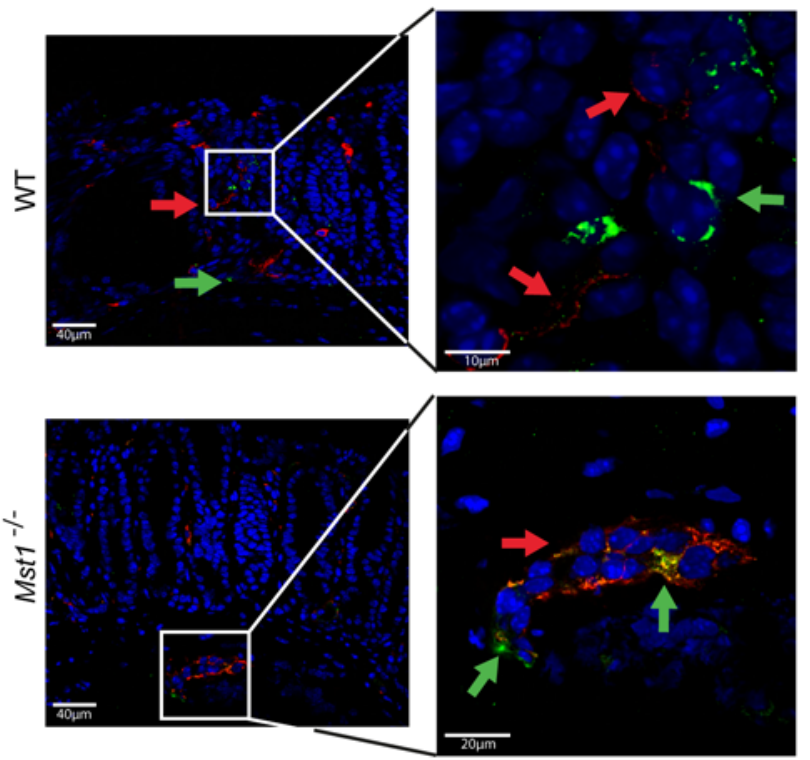

B

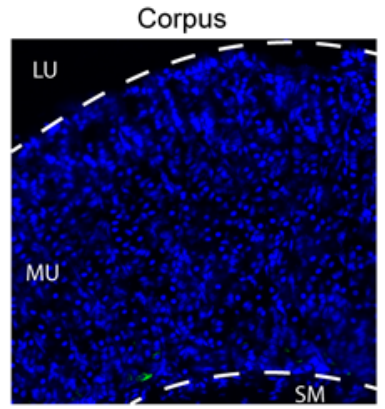

MU

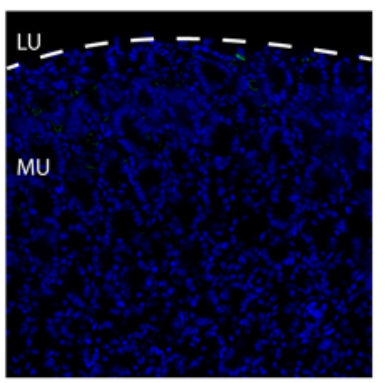

E

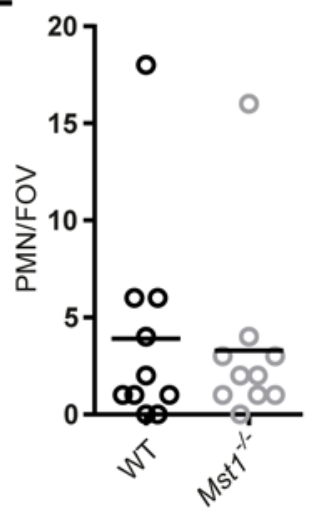

C
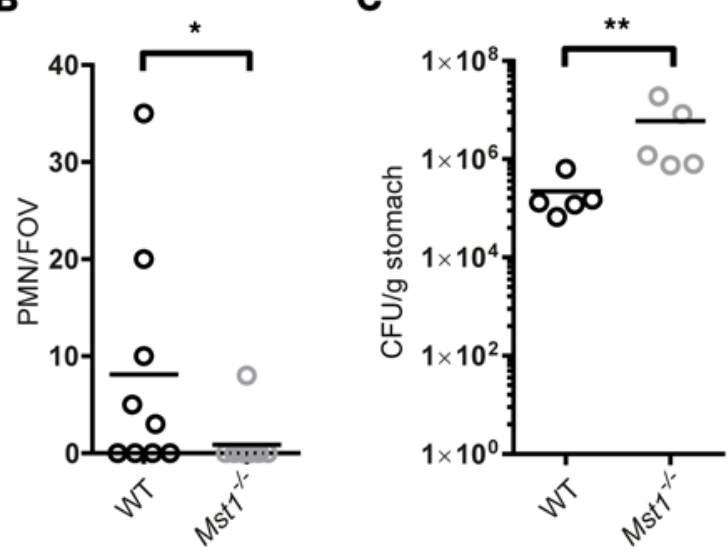

$\mathbf{F}$

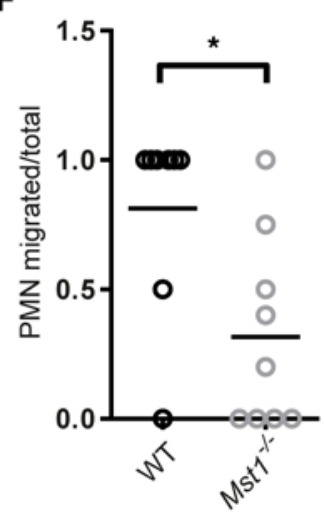

Figure 3. Mst1/- neutrophils fail to extravasate and migrate to the site of infection in an $\mathbf{H}$. pylori mouse infection model. (A) Representative images are shown of the antrum and corpus from WT and Mst1 ${ }^{--}$mice 3 months after $\mathrm{H}$. pylori infection. LU, lumen; MU, mucosa; SM, submucosa. Neutrophils were identified with Ly6C antibody (green). Nuclei are visualized with DAPI (blue). Scale bar: $10 \mu \mathrm{m}$. (B) Number of transmigrated neutrophils per field of view (FOV) in the antrum of WT and Mst1 ${ }^{-1-}$ mice is displayed ( $n=9$ mice, scatter plot with mean, ${ }^{*} P<0.05$, Mann-Whitney test). (C) The CFUs per gram stomach of WT and Mst1 ${ }^{-1-}$ mice were evaluated 6 weeks after infection ( $n=5$ mice, scatter plot with mean, ${ }^{* *} P<0.01$, Mann-Whitney test). (D) Immunostaining of the antrum of WT and Mst1 ${ }^{-1-}$ mice for PECAM-1 (red) and Ly6G antibody (green). Nuclei are visualized with DAPI (blue). Scale bar: 40 $\mu \mathrm{m}$. Close-ups display high magnification of blood vessel-rich areas. Red arrows indicate blood vessels; green arrows indicate neutrophils. Scale bar: $10 \mu \mathrm{m}$ (top), $20 \mu \mathrm{m}$ (bottom). (E) Number of neutrophils per field of view from WT and Mst1-- mice ( $n=10$ mice, scatter plot with mean, NS, Mann-Whitney test). (F) Ratio of extravasated neutrophils to total number of neutrophils is depicted from WT and $M s t 1^{-/-}$mice $\left(n=10\right.$ mice, scatter plots with mean, ${ }^{*} P<0.05$, Mann-Whitney test).

within the 20 minutes of observation $(20 \pm 0.8$ minutes, median \pm SEM) (Figure 2B) and accumulated at the abluminal site of postcapillary venules (Figure 2C).

To test whether further inflammatory stimulation could induce migration into the interstitial matrix, a laser injury near postcapillary venules was set 3 hours after TNF- $\alpha$ stimulation. Neutrophils of $L y z 2^{G F P}$ mice swarmed and accumulated at the site of laser injury within 30 minutes, as described by Lämmermann et al. (37). Interestingly, neutrophils deficient in MST1 remained at the abluminal site of venules and were unable to interstitially migrate to the site of laser injury (Figure 2, D and E, and Supplemental Videos 5 and 6). However, absence of interstitial migration was not due to an intrinsic migration defect of $\mathrm{Mst1}^{-/-}$neutrophils, as migration of bone marrow-derived neutrophils to fMLP or CXCL1 in a 3D collagen gel assay was similar to that of WT neutrophils (Supplemental Figure 2, A and B). 
A
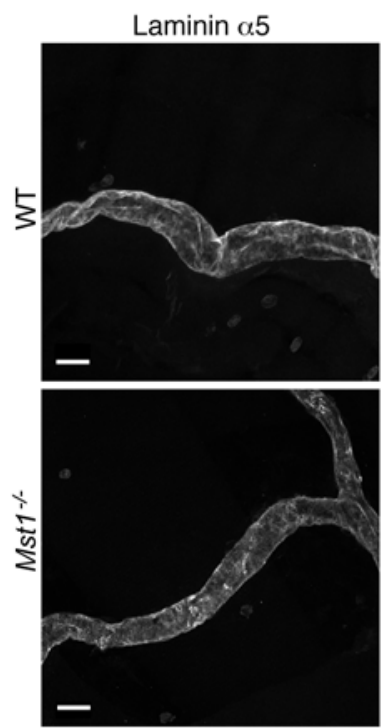

B
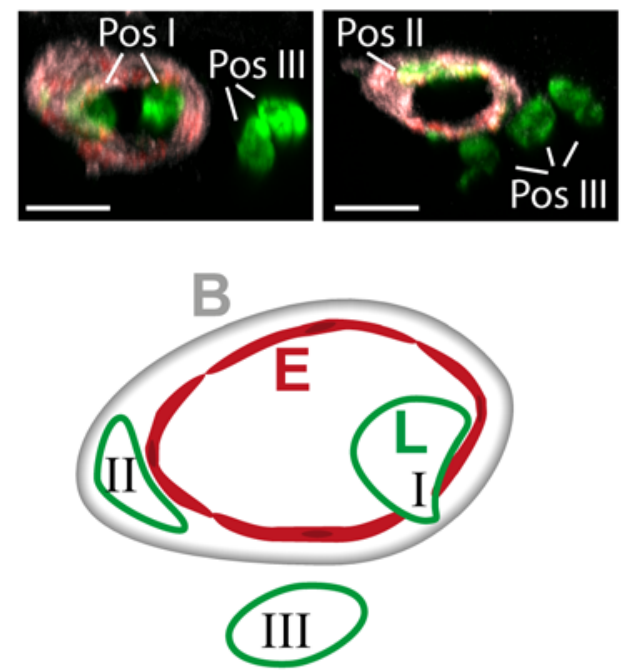

D

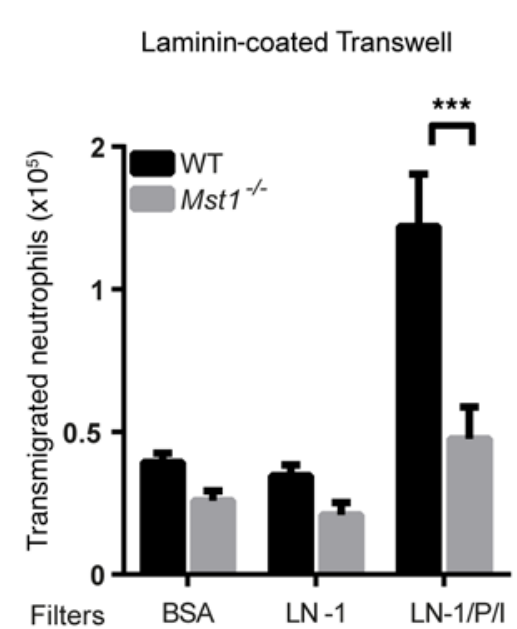

CXCL1

ESAM1 Pos III
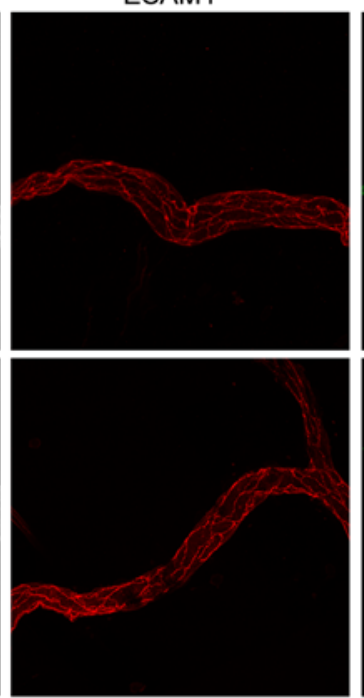

C

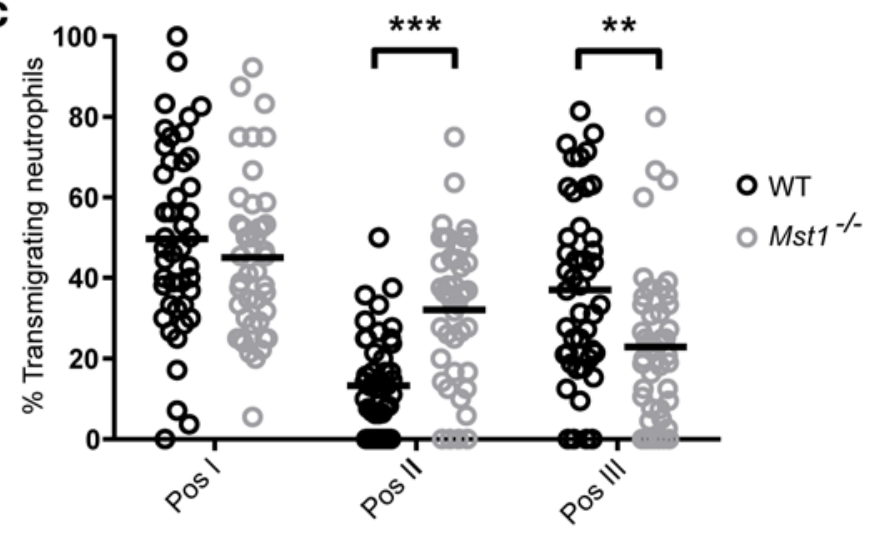

MRP14
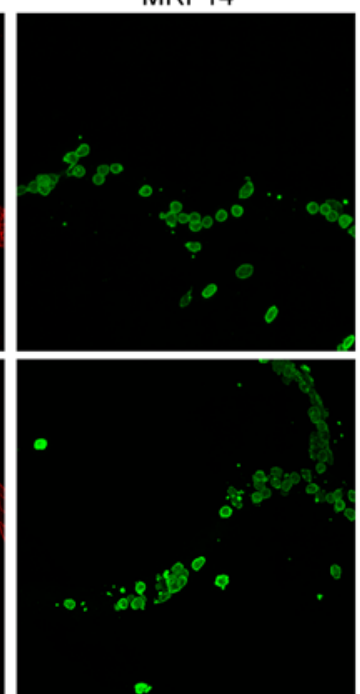

Merge

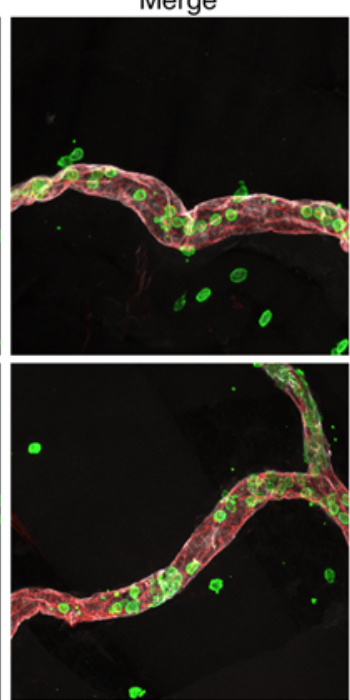

E

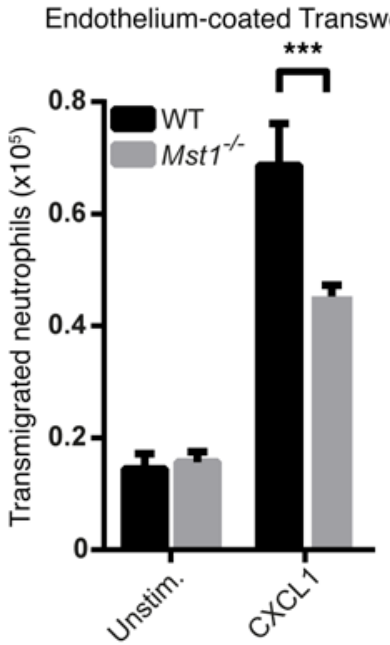

$\mathbf{F}$

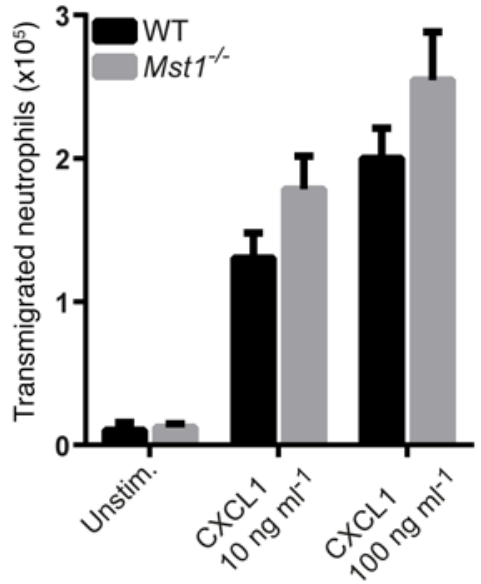


Figure 4. Mst1 ${ }^{-/-}$neutrophils fail to penetrate the BM in vivo and in vitro. (A) Immunostaining of TNF- $\alpha$-stimulated cremaster muscle whole mounts from WT and Mst1 ${ }^{-1-}$ mice for laminin $\alpha 5$ (white, BM), ESAM1 (red, endothelial junctions), and MRP14 (green, neutrophils) $(n=3)$. Scale bar: $20 \mu \mathrm{m}$. (B) $3 \mathrm{D}$ reconstruction and illustration of a vessel cross section demonstrating the classification of transmigrating neutrophils respective to their position. Position I, neutrophils in intimate contact with the endothelium; position II, neutrophils between the endothelium and the BM; position III, fully transmigrated neutrophils. Scale bar: $10 \mu \mathrm{m}$. (C) Distribution pattern of transmigrating neutrophils respective to their positions (positions I-III) in WT and Mst1 ${ }^{-/-}$ mice ( $n=45$ analyzed vessels from 5 mice per group, scatter plot with mean of $>25$ analyzed vessels per group, ${ }^{* * *} P<0.001,{ }^{* *} P<0.01,2$-way ANOVA, Sidak's multiple comparisons test). (D-F) Neutrophil transmigration in a Transwell assay with or without CXCL1 through filters coated with BSA (control), Iaminin 1 (LN-1), or LN-1, PECAM-1, and ICAM-1 (LN-1/P/I) ( $n=3$ mice, triplicate measurements were performed, mean \pm SEM, ${ }^{* * *} P<0.001,2$-way ANOVA, Sidak's multiple comparisons test) (D); with or without CXCL1 through an endothelial monolayer of 1G11 cells $(n=3$ mice, triplicate measurements were performed, mean \pm SEM, ${ }^{* * *} P<0.001$, 2-way ANOVA, Sidak's multiple comparisons test) (E); and to HBSS, $10 \mathrm{ng} / \mathrm{ml}$ and $100 \mathrm{ng} / \mathrm{ml} \mathrm{CXCL}$ ( $n=3 \mathrm{mice}$, duplicate measurements were performed, mean \pm SEM, 2-way ANOVA, Sidak's multiple comparisons test) (F).

MST1-deficient neutrophils fail to transmigrate to the site of infection in a Helicobacter pylori mouse infection model. Next, we evaluated the role of MST1 in a murine Helicobacter pylori infection model. Previous work had shown that neutrophils play an important role in the pathogenesis of H.pylori infection (38). The bacterium induces a strong inflammatory response in the gastric mucosa, which is dependent on the $H$. pylori neutrophil-activating protein (HP-NAP). Furthermore, proinflammatory cytokines such as CXCL8 are secreted during the inflammatory process, recruiting additional neutrophils and monocytes to the site of inflammation (39).

We infected WT and $M s t 1^{-/-}$mice with $H$. pylori orogastrically and sacrificed the mice 3 months later. As reported earlier (40), in WT mice, the majority of infiltrated neutrophils were found in the antrum region of the stomach (Figure $3 \mathrm{~A}$, top panel). In contrast, we only detected a very small number of neutrophils in the gastric epithelium of the antrum of $\mathrm{Mst1}^{-/-}$mice (Figure 3A, bottom panel), demonstrating reduced neutrophil extravasation (Figure 3B). In line with this, the number of CFU of $H$.pylori in the antrum showed a significant increase in $\mathrm{Mst1}^{-/-}$mice compared with WT mice (Figure 3C), indicating a more successful colonization.

Finally, we immunostained tissue sections from the lower part of the mucosa, where neutrophils extravasate from postcapillary venules (Figure 3D). We did not observe any difference in the number of total neutrophils per field of view between WT and $\mathrm{Mst1}^{-1-}$ mice (Figure 3E). However, in WT mice neutrophils migrated from venules into the inflamed tissue, while, as expected, neutrophils from $\mathrm{Mst1}^{-/-}$mice remained near the vessel wall (Figure 3F). Thus, MST1 is required for proper neutrophil extravasation from the submucosal vasculature into tissue during $H$. pylori infection.

MST1 is essential for neutrophils to penetrate the BM. Next, we focused on the capacity of $\mathrm{Mst1}^{-{ }^{-}}$neutrophils to penetrate the perivascular BM. To this end, we performed whole-mount stainings of TNF- $\alpha$-stimulated cremaster muscles using antibodies against laminin $\alpha 5$, endothelial cell-specific adhesion molecule 1 (ESAM1), and MRP14 to visualize the BM, endothelial cell contacts, and neutrophils, respectively (Figure 4A). By means of confocal microscopy, neutrophils were classified into 3 groups according to their position during the extravasation from postcapillary venules. Neutrophils embedded within the endothelial layer belonged to position I. Position II represented neutrophils located between the endothelium and BM, and fully transmigrated neutrophils were classified as being in position III (Figure 4B) (7). Neutrophils of WT and $M s t 1^{-/-}$mice were present at similar percentages embedded within the endothelium (position I). However, significantly more $\mathrm{Mst1}^{-/-}$neutrophils were located between the endothelium and the BM (position II) compared with WT neutrophils, while significantly more WT than $\mathrm{Mst1}^{-/-}$neutrophils were fully transmigrated (position III) (Figure 4C).

To gain further insights into the molecular mechanisms of MST1-dependent transmigration, we performed Transwell assays where filters were coated with BSA (control); with laminin, a key component of the BM, alone (41); or with PECAM-1 and ICAM-1; while CXCL1 was used as chemoattractant. Laminin alone did not induce transmigration of either WT nor $\mathrm{Mst1}^{-/-}$ neutrophils. A combination of laminin, PECAM-1, and ICAM-1 efficiently induced transmigration along a CXCL1 gradient, but only for WT, not $\mathrm{Mst1}^{-/}$, neutrophils (Figure 4D). In line with this, $M s t 1^{-/-}$neutrophils failed to transmigrate as efficiently as WT neutrophils through filters containing a monolayer of mouse endothelial 1G11 cells in response to CXCL1 (Figure 4E). Importantly, we did not find differences in transmigrated WT and $M s t 1^{-/-}$neutrophils in Transwell assays without coating (Figure $4 \mathrm{~F}$ ), implying that MST1 is essential for neutrophils to penetrate the $\mathrm{BM}$ in vivo and in vitro.

$M S T 1$ is required for the translocation of VLA-3,VLA-6, and NE to the cell surface. It had been shown previously that mobilization of VLA-3, VLA-6, and NE from intracellular stores to the plasma membrane is a crucial step for successful neutrophil transmigration $(14,16,17)$. In order to evaluate the putative role of MST1 in the mobilization of VLA-3, VLA-6, and NE, neutrophils from WT or $\mathrm{Mst1}^{-/-}$mice were seeded on slides coated with BSA or PECAM-1/ICAM-1/CXCL1 and immunostained with antibodies against VLA-3, VLA-6, or NE. Confocal microscopy showed that WT as well as $\mathrm{Mst1}^{-/-}$neutrophils seeded on BSA displayed all 3 molecules - VLA-3, VLA-6, and NE (Figure 5, A, D, and G) within intracellular stores. Fluorescence intensity profiles (see Methods and Supplemental Figure 3A) of VLA-3, VLA-6, and NE were similar between WT and $\mathrm{Mst1}^{-/-}$cells seeded on BSA (Supplemental Figure 3, B-D). PECAM-1/ICAM-1/CXCL1 coating induced the translocation of VLA-3, VLA-6, and NE to the surface of interacting WT neutrophils, leading to a ring-like structure at the cell border (Figure 5, A-I). In contrast, neutrophils derived from $M s t 1^{-/-}$mice failed to efficiently mobilize any of the 3 proteins to the cell surface, resulting in less pronounced ring formation in $\mathrm{Mst1}^{-/-}$neutrophils (Figure 5, A-I). Notably, Western blot analysis showed no difference in the total protein levels of VLA-3, VLA-6, and NE between WT and $\mathrm{Mst1}^{-/-}$neutrophils (Supplemental Figure 3E).

In order to verify MST1-dependent mobilization in vivo, we investigated NE activity with an NE-fluorescent activatable substrate (NE680FAST) in postcapillary venules of unstimulated 
A

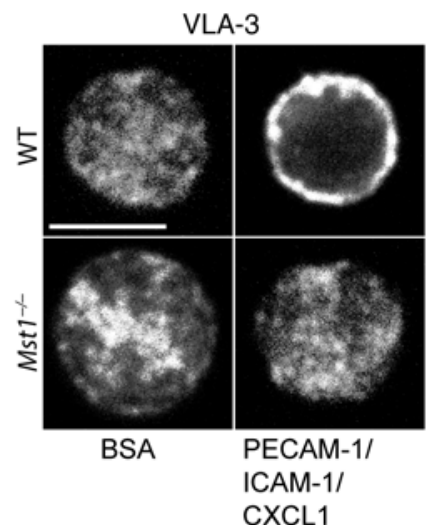

D

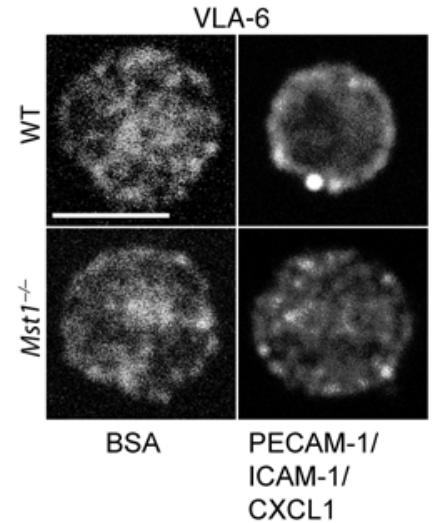

G

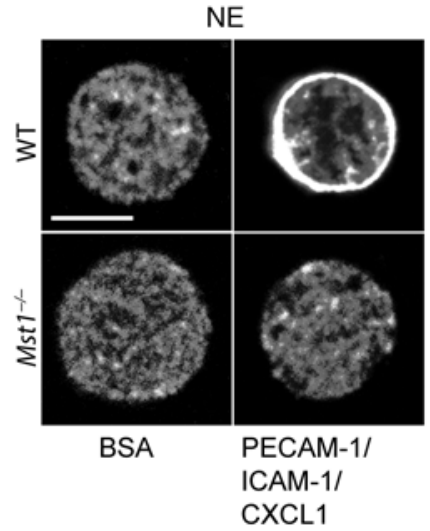

B

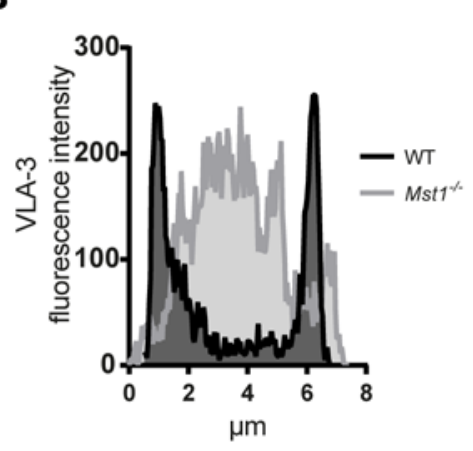

E

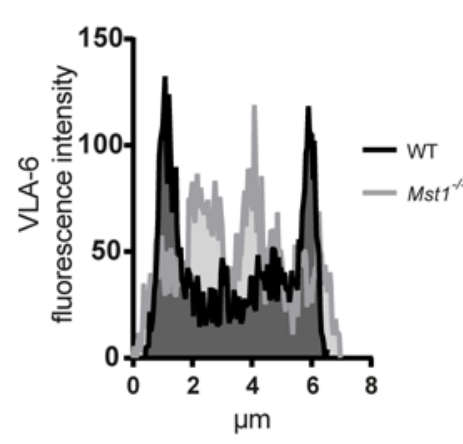

H

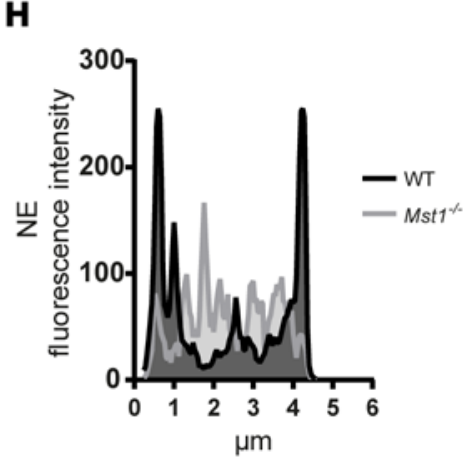

C

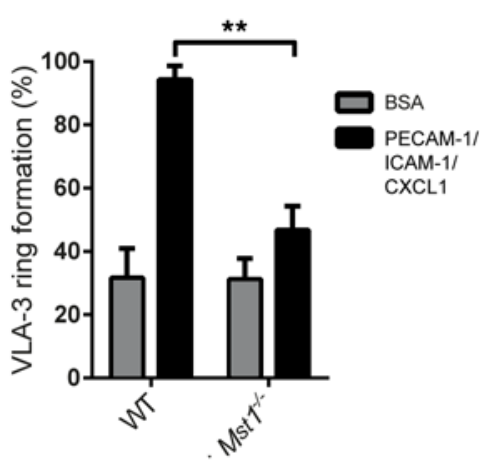

$\mathbf{F}$

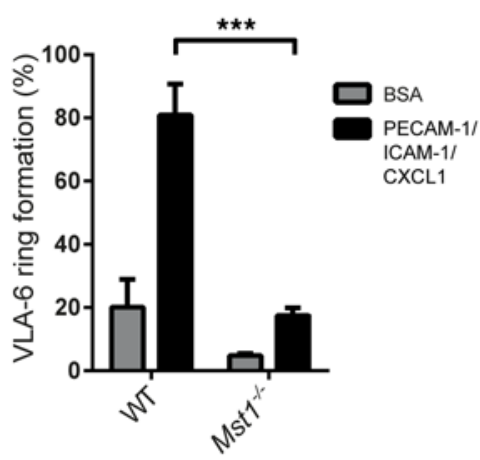

I

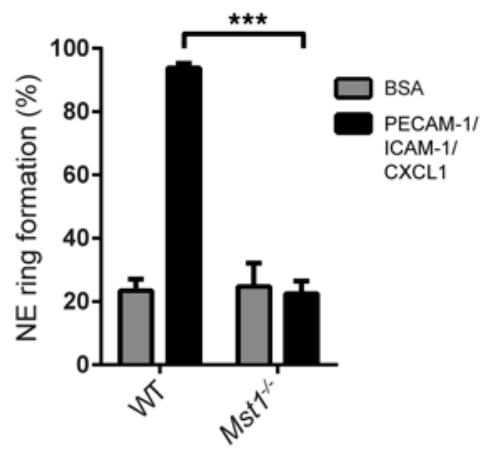

J
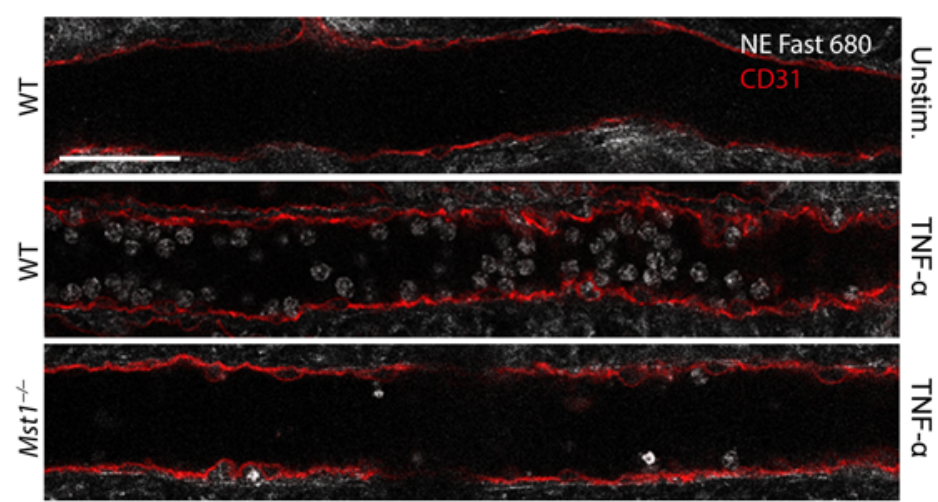

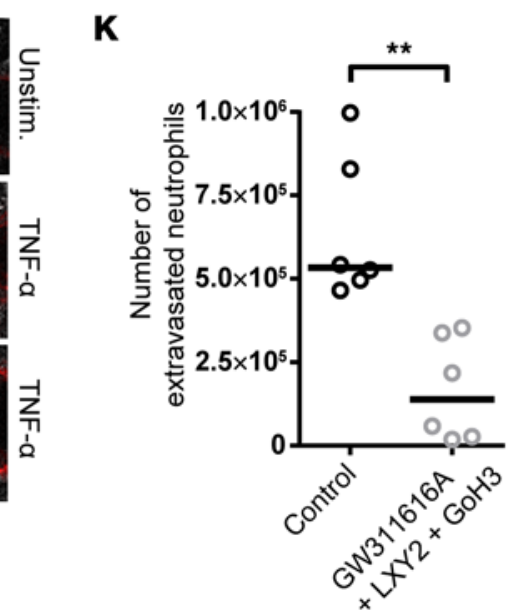


Figure 5. MST1 is required for the mobilization of VLA-3, VLA-6, and NE to the plasma membrane. (A, D, and G) Immunostaining of representative WT and $\mathrm{Mst1}^{-/-}$neutrophil on BSA- or PECAM-1/ICAM-1/CXCL1-coated wells for VLA-3 (A), VLA-6 (D), and NE (C). Scale bar: $5 \mu \mathrm{m}$. (B, E, and H) Fluorescence intensity profiles along a line through the cell for VLA-3 (B), VLA-6 (E), and NE (H) of a representative WT (black line) and Mst $7^{-1-}$ neutrophil (gray line) seeded on PECAM-1/ICAM-1/CXCL1-coated wells. (C, F, and I) Quantification of ring formation of VLA-3 (C), VLA-6 (F), and NE (I) ( $n>50$ analyzed neutrophils from 3 mice per group, mean $\pm \mathrm{SEM},{ }^{* *} P<0.01$, ${ }^{* *} P<0.001$, 2-way ANOVA, Sidak's multiple comparisons test or Tukey's multiple comparisons test). (J) NE activity (white) within venules in unstimulated and TNF- $\alpha$-stimulated cremaster muscle whole mounts from WT and Mst1 ${ }^{-1-}$ mice imaged by confocal microscopy. Venules were visualized using a rat anti-mouse PECAM- 1 antibody (red) ( $n=3$ mice per group). Scale bar: $50 \mu \mathrm{m}$. (K) Total number of extravasated neutrophils in the peritoneal lavage 2 hours after i.p. injection of TNF- $\alpha$ in WT mice after treatment with either the VLA-3-blocking peptide LXY2, the $\alpha 6$-blocking mAb GoH3, and the NE inhibitor GW311616A; or solvent alone, control peptide, and isotype-matched control mAb ( $n=6$, mean \pm SEM, ${ }^{* *} P<0.01$, unpaired $t$ test).

and TNF- $\alpha$-stimulated cremaster muscle whole mounts from WT and $M s t 1^{-/-}$mice. In unstimulated venules of WT mice we were unable to detect any NE activity, while 2 hours after TNF- $\alpha$ stimulation NE activity could be observed (Figure 5J). TNF- $\alpha$-stimulated venules from $\mathrm{Mst1}^{-/-}$mice showed decreased $\mathrm{NE}$ activity as compared with those from WT mice (Figure 5J). Importantly, the number of adherent cells was similar in WT and $\mathrm{Mst1}^{-/-}$mice (Supplemental Figure 3F).

In order to confirm that blocking VLA-3, VLA-6, and NE affects neutrophil extravasation in vivo we used the TNF- $\alpha-$ induced peritonitis model. WT mice treated with the VLA-3blocking peptide LXY2, the $\alpha 6$-blocking $\mathrm{mAb}$ GoH3, and the NE inhibitor GW311616A before i.p. injections of TNF- $\alpha$ displayed a decreased number of transmigrated neutrophils compared with control WT mice (Figure 5K).

Taken together, these in vitro and in vivo results demonstrate that MST1 is indispensable for efficient translocation of VLA-3, VLA-6, and NE to the surface of neutrophils.

MST1 regulates vesicle trafficking together with JFC1, a Rab27 effector molecule. Rab27a is a central regulator of vesicle trafficking in neutrophils and has been shown to influence many neutrophil functions through its ability to regulate vesicle exocytosis $(21,42)$. Therefore, we investigated Rab27a translocation to the plasma membrane in response to PECAM-1/ICAM-1/ CXCL1 stimulation. Rab27a displayed an intracellular localization in WT and $\mathrm{Mst1}^{-/-}$neutrophils when seeded on BSA (Figure 6A and Supplemental Figure 4A). In WT neutrophils, PECAM-1/ ICAM-1/CXCL1 coating induced the translocation of Rab27a to the plasma membrane (Figure 6, A and B) and led to ring formation of Rab27a in most analyzed WT neutrophils (Figure 6C). In contrast, neutrophils derived from $\mathrm{Mst1}^{-/-}$mice failed to translocate Rab27a to the plasma membrane in response to PECAM-1/ ICAM-1/CXCL1 stimulation (Figure 6, A-C). Importantly, total protein levels of Rab27a were similar between WT and $\mathrm{Mst1}^{-1}$ neutrophils (Supplemental Figure 4B).

Next, we investigated whether MST1 itself translocates to the plasma membrane upon ICAM-1/PECAM-1/CXCL1 stimulation. MST1 displayed intracellular localization in WT neutrophils seeded on BSA (Figure 6, D-F). In response to ICAM-1/PECAM-1/ CXCL1 stimulation, MST1 localized together with Rab27a at the plasma membrane (Figure 6, D-F).

Finally, we examined the role of Rab27a and its 2 effector molecules, JFC1 and Munc13-4 (22-25), during VLA-3 and VLA6 translocation. To do this, we investigated the mobilization of VLA-3 and VLA- 6 in response to PECAM-1/ICAM-1/CXCL1 in WT, Rab27aash/ash (Rab27a knockout), Sytl1-/- (JFC1 knockout), and Munc13-4 $4^{i n x} / /$ inx $x$ (Munc13-4 knockout) neutrophils. In WT,
Rab27a $a^{\text {ash/ash }}$, and Munc13-4 $4^{\text {inx } x / \text { inx }}$ neutrophils, PECAM-1/ICAM-1/ CXCL1 coating induced the translocation of VLA-3 to the plasma membrane. In contrast, neutrophils derived from $\mathrm{Sytl}^{-1-}$ mice showed decreased translocation of VLA-3 (Figure 6G). Furthermore, translocation of VLA- 6 was decreased in neutrophils from Sytl1 ${ }^{-/}$mice compared with neutrophils of WT and Rab27a ash/ash mice. Interestingly, neutrophils from Munc13-4 $4^{\text {inx } x / j n x}$ mice showed an increased translocation of VLA-6 compared with WT neutrophils (Figure 6H).

Next, we used a continuous sucrose gradient and demonstrated that Rab27a and MST1 are present in the same low-density granule fraction of human neutrophils (Figure 6I), suggesting that the interaction of these proteins contributes to the effective transport of VLA-3- and VLA-6-containing vesicles to the neutrophil surface.

Taken together, our results show that MST1 and Rab27a are present in, and are located in the same subpopulation of, neutrophil granules. Furthermore, we provide evidence that MST1 regulates PECAM-1/ICAM-1/CXCL1-stimulated trafficking of vesicles containing VLA-3 and VLA- 6 to the neutrophil surface via cooperation with the Rab27 effector protein JFC1, but not Munc13-4.

\section{Discussion}

In this study, we identify the serine/threonine kinase MST1 as the first signaling molecule indispensable for neutrophil BM penetration into inflamed tissue.

Originally, MST1 (mammalian homolog of the Drosophila Hippo protein) was identified in D. melanogaster as the central constituent of the Hippo pathway, controlling organ size and proliferation. Recently, MST1 has attracted more attention due to its role in cancer development (43) and the discovery that a mutation in human STK4 (MST1) leads to a primary immune deficiency. Patients with a deficiency in STK4 present with recurring bacterial, fungal, and viral infections, and display lymphopenia and intermittent neutropenia (26-28). Using $\mathrm{Mst1}^{-/-}$mice, MST1 was shown to be required for LFA-1-dependent T cell trafficking (31) by regulating the transport and distribution of LFA-1-containing vesicles to the plasma membrane and subsequent LFA-1 clustering and adhesion of $\mathrm{T}$ cells (30).

We demonstrate that - in contrast to T cells - MST1 is dispensable for LFA-1-dependent neutrophil adhesion in mice and humans. Neutrophils from $M s t 1^{-/}$mice showed no defect in LFA-1 activation. In addition, clustering of LFA-1 as well as static adhesion on ICAM-1-coated surfaces was not altered in $\mathrm{Mst1}^{-/ /}$neutrophils compared with WT neutrophils. Murine $\mathrm{Mst1}^{-/-}$neutrophils as well as neutrophils derived from 2 patients with STK 4 deficiency adhered to E-selectin/ICAM-1/CXCL1-coated flow chambers 
A

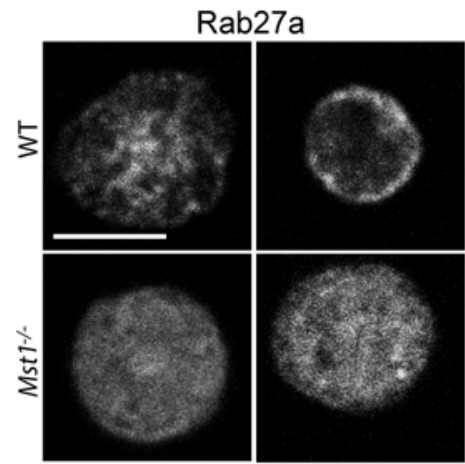

BSA

PECAM-1/

ICAM-1/

D

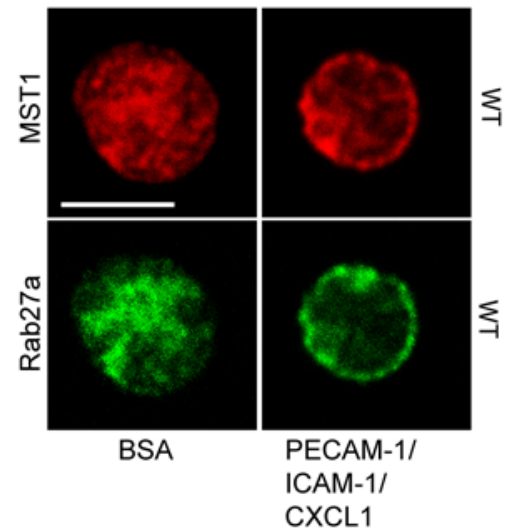

G

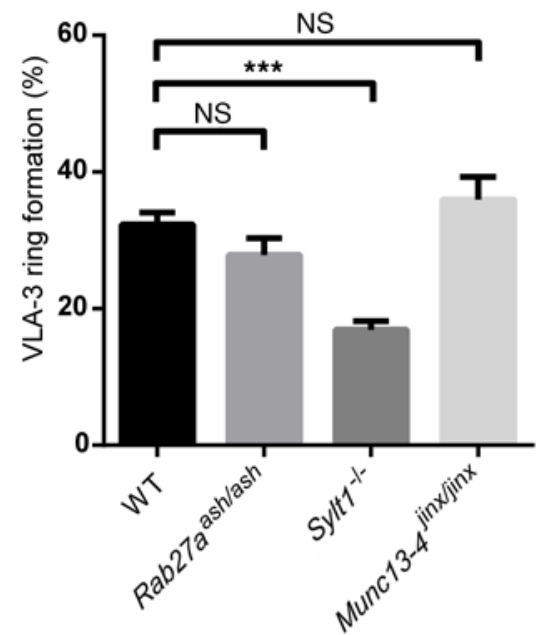

B

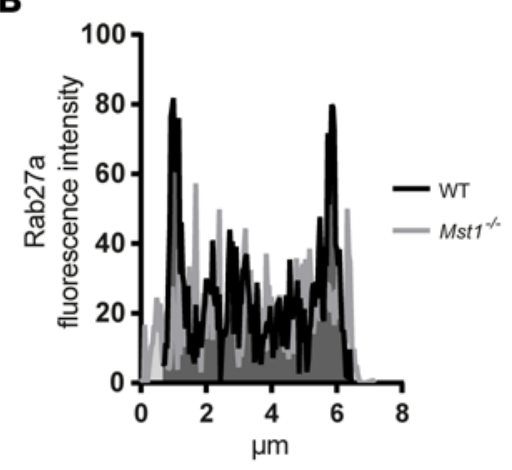

E

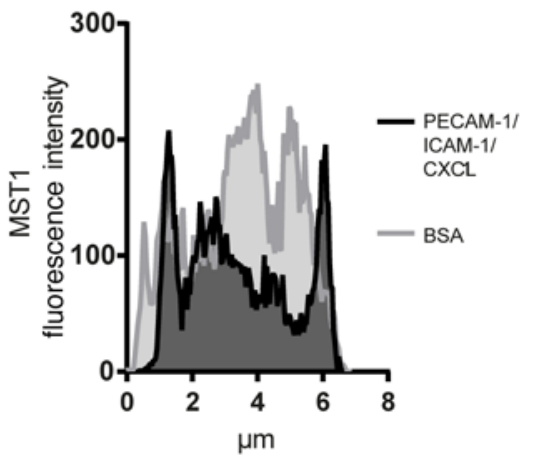

H

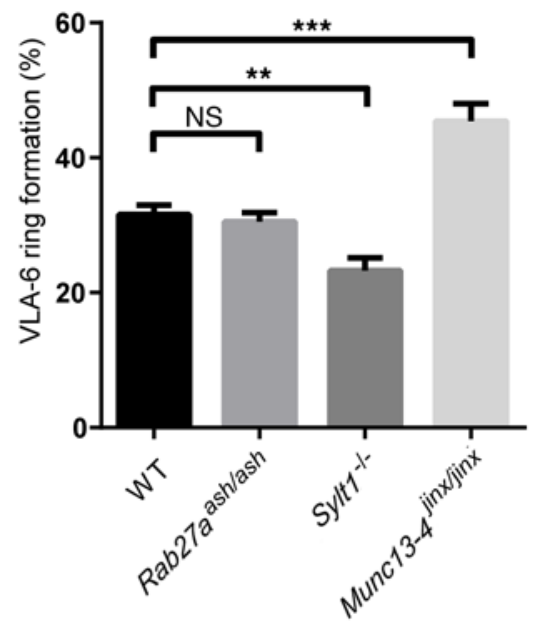

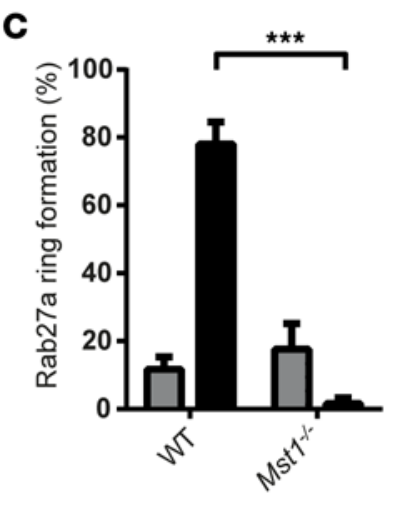

F

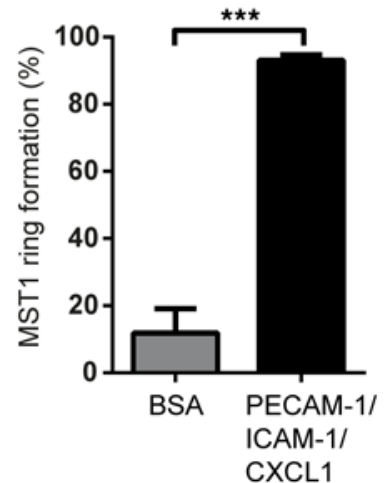

I

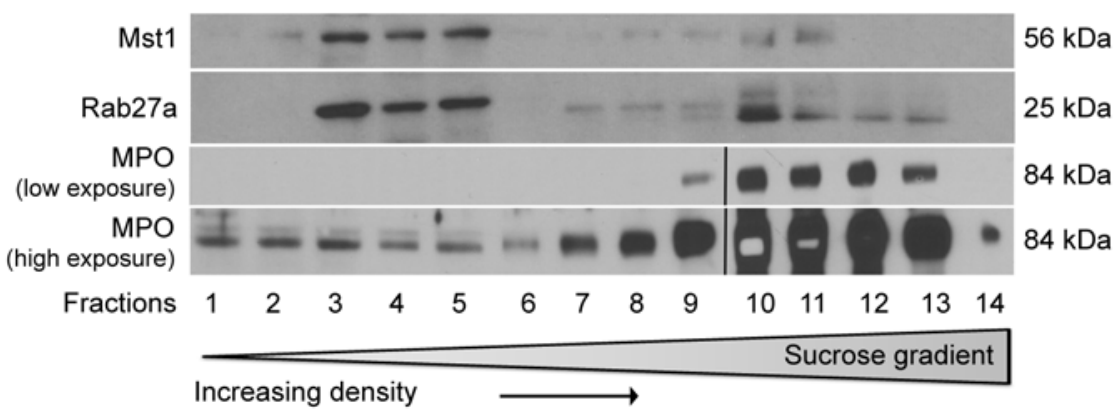


Figure 6. MST1 cooperates with JFC1 and induces translocation of Rab27a-positive vesicles to the plasma membrane. (A and D) Immunostaining of WT and Mst1 ${ }^{-1-}$ neutrophils seeded on BSA- or PECAM-1/ICAM-1/CXCL1-coated wells for Rab27a (A) and Rab27a (green) and MST1 (red) (D). Scale bar: $5 \mu$ m. (B and E) Fluorescence intensity profiles assessed along a line through the cell for Rab27a of representative WT (black line) and Mst1 ${ }^{-1-}$ neutrophil (gray line) seeded on PECAM-1/ICAM-1/CXCL1-coated wells (B) and for MST1 of representative WT neutrophil seeded on BSA-coated (gray line) or PECAM-1/ICAM-1/CXCL1-Coated wells (black line) (E). (C and F) Quantification of ring-like expression of Rab27a ( $n>50$ analyzed neutrophils from 3 mice per group, mean \pm SEM, unpaired $t$ test) (C) and MST1 in response to BSA or PECAM-1/ICAM-1/CXCL1 stimulation ( $n \geq 50$ analyzed neutrophils from 3 mice per group, mean \pm SEM, 2-way ANOVA, Sidak's multiple comparisons test) (F). (G and $\mathbf{H})$ Quantification of ring formation of neutrophils from WT, Rab27a ash/ash, Syt/1/--, and Munc13-4inx/inx mice seeded on PECAM-1/ICAM-1/CXCL1-coated wells for VLA-3 (G) and VLA-6 (H) $(n>900$ analyzed cells from $\geq 3$ mice per group, mean \pm SEM, 1-way ANOVA, Dunnett's multiple comparisons test). (I) Neutrophils were disrupted and fractionated using a continuous sucrose gradient. Samples were analyzed by Western blot for the presence of MST1 and Rab27a, and for the presence of myeloperoxidase (MPO), a marker of azurophilic granules. Lanes were run in different gels, developed simultaneously using the same development solution and same exposure time (black lines). ${ }^{* *} P<0.01,{ }^{* * *} P<0.001$.

to the same extent as WT controls or the patients' heterozygote parents, respectively. These results suggest that different mechanisms of LFA-1 regulation/activation exist between lymphocytes and neutrophils. This may be supported by data from Fabbri et al., reporting that Rab11 is required for the trafficking of LFA-1-containing vesicles in neutrophils (44). In T cells, Rab13 is responsible for this step (29).

Although $\mathrm{Mst1}^{-{ }^{-}}$neutrophils showed no defective adhesion in our studies, they failed to extravasate into the peritoneal cavity. In TNF- $\alpha$-stimulated cremaster muscles, $\mathrm{Mst1}^{-/}$neutrophils remained in close contact with the abluminal side of the vessel. Even after additional stimulation by induction of a laser injury next to the vessel, $\mathrm{Mst1}^{-/}$neutrophils persisted adjacent to the vessel wall and failed to migrate to the site of injury. After crossing the endothelial cell layer, neutrophils have to penetrate the underlying $\mathrm{BM}(3,5,13)$. The $\mathrm{BM}$ of postcapillary venules is mainly composed of laminin 411 ( $\alpha 4 \beta 1 \gamma 1$ laminin subunit), laminin 511 ( $\alpha 5 \beta 1 \gamma 1$ laminin subunit), and type IV collagen (5). Although many studies have been performed on leukocyte adhesion and transendothelial migration, the mechanism by which neutrophils penetrate the BM is still poorly understood $(3,4)$.

Previous studies showed that genetic, antibody- or inhibitor-mediated blockade of PECAM-1 (17), VLA-3 (14, 16), VLA-6 $(15,17,45)$, or NE (17) leads to an arrest of infiltrating neutrophils between the endothelial layer and the underlying BM. Extravasating neutrophils need to interact with ICAM-1 and PECAM-1 to induce the mobilization of vesicles containing VLA- 6 and NE. This mobilization translocates VLA- 6 and NE to the cellular surface, a prerequisite to pass the BM $(17,45)$. We found that MST1 is essential for the exocytosis of VLA-3, VLA-6, and NE, as neutrophils from $\mathrm{Mst1}^{-/-}$mice failed to translocate these proteins to the cell surface upon stimulation with PECAM-1/ICAM-1/CXCL1. In contrast to WT neutrophils, neutrophils from $\mathrm{Mst1}^{-/-}$mice failed to pass through a PECAM-1-, ICAM-1-, and laminin-coated Transwell filter in an in vitro transmigration assay. Furthermore, blockade of VLA-3, VLA-6, and NE decreased neutrophil extravasation into the peritoneal cavity in WT mice in response to TNF- $\alpha$.

As MST1 influences LFA-1 clustering in T cells by regulating LFA-1-containing vesicle trafficking via Rab13 (29), we hypothesized that MST1 is similarly involved in the mobilization of the vesicles containing VLA-3, VLA-6, and NE to the plasma membrane in neutrophils. The GTPase Rab27a is a master regulator of exocytosis in neutrophils (21). Several studies indicate that Rab27a and its effectors JFC1 and Munc13-4 regulate the secretion of azurophilic granules containing $\mathrm{NE}$ and myeloperoxidase $(22,24)$. We discovered that Rab27a and MST1 themselves translocate to the plasma membrane in response to PECAM-1/ ICAM-1/CXCL1. However, translocation of Rab27a is defective in $\mathrm{Mst1}^{-/}$neutrophils, indicating that MST1 regulates Rab27a-positive vesicle trafficking. Additional studies of VLA-3 and VLA- 6 mobilization performed using primary neutrophils from Rab27a knockout mice (Rab27 $a^{a s h} /$ ssh) show that in the absence of Rab27a the mobilization of these markers is only partially decreased and not significantly different from the phenotype observed in control cells. This can be explained by a possible compensatory mechanism mediated by the small GTPase Rab27b. In fact, although Rab27a is the predominant Rab27 GTPase in neutrophils (46), neutrophils lacking Rab27a show increased levels of Rab27b (46), a protein that shares $71 \%$ homology with Rab27a at the amino acid level (47). Rab27b has functional redundancy with Rab27a (47) and is known to mediate many common trafficking mechanisms through interaction with Rab27-specific effectors (48, 49). In order to better understand the mechanism underlying the regulation of VLA-3 and VLA- 6 mobilization and to establish a definite role for Rab27a-dependent mechanisms in this process, we next studied neutrophils from mice lacking the expression of the Rab27 effector JFC1 (50) or Munc13-4 (51), 2 molecules known to bind to and modulate processes regulated by both Rab27a $(50,51)$ and Rab27b $(48,49)$. Our data demonstrate that Sytl1 ${ }^{-/}$neutrophils have a significant impairment in the mobilization of VLA-3 and VLA-6, thus mimicking the effect observed in $\mathrm{Mst1}^{-/}$neutrophils. These data, together with the observations that MST1 cofractionates with Rab27a (and JFC1; ref. 50), suggest that MST1 regulates the mobilization of Rab27a-positive vesicles together with the specific effector JFC1. Our results also demonstrate that in Munc13-4-deficient neutrophils, the mobilization of VLA-3 and VLA- 6 is not impaired. In fact, a significant increase in this process was observed in the absence of Munc13-4. This can be explained by a putative mechanism of competition between Munc13-4 and JFC1 for Rab27a and a consequent increase of the interaction between JFC1 and Rab27a in the absence of Munc134. Altogether, our data strongly support a role for MST1 and the Rab27a effector JFC1 in the mobilization of both VLA-3- and VLA-6- and Rab27a-positive vesicles.

In conclusion, we demonstrate that the serine/threonine kinase MST1 is indispensable for neutrophil penetration of the vascular BM by regulating the mobilization of VLA-3, VLA-6, and NE from intracellular vesicles to the plasma membrane. These findings elucidate the importance of MST1 for early innate immune responses. They also show that this neutrophil transmigration defect contributes to the phenotype of recurring infections found in patients suffering from STK 4 immune deficiency. 


\section{Methods}

Western blot. Isolated bone marrow-derived neutrophils (EasySep mouse neutrophil enrichment kit; STEMCELL Technologies) were incubated in lysis buffer $(150 \mathrm{mM} \mathrm{NaCl}, 1 \%$ Triton X-100 [Applichem], $0.5 \% \mathrm{Na}$ deoxycholate [Sigma-Aldrich], $50 \mathrm{mM}$ Tris- $\mathrm{HCl}$ pH 7.3 [Merck], 2 mM EDTA [Merck] supplemented with protease [Roche] and phosphatase inhibitors [Sigma-Aldrich]), homogenized in Laemmli sample buffer, and boiled for 5 minutes. Proteins were resolved by SDS-PAGE and then electrophoretically transferred onto PVDF membranes, which were subsequently incubated with antibodies. The following antibodies were used: rabbit anti-MST1 antibody (Upstate), rabbit anti-MST2 antibody (Cell Signaling Technology), rabbit anti-NE antibody (Abcam), mouse anti-VLA-3 antibody (42/ CD49c; BD), rabbit anti-VLA-6 antibody (Thermo Scientific), sheep anti-Rab27a (R\&D Systems), mouse anti-GAPDH antibody (Calbiochem), goat anti-mouse-HRP, goat anti-rabbit-HRP, or donkey anti-sheep-HRP (Jackson ImmunoResearch). Bound antibodies were detected using ECL (Millipore Corp.).

Intravital microscopy of TNF- $\alpha$-stimulated mouse cremaster muscle venules. Intravital microscopy of mouse cremaster muscle venules was performed in WT and $\mathrm{Mst1}^{-/-}$mice, as previously described (52). Briefly, 2 hours after intrascrotal injection of TNF- $\alpha$ (R\&D Systems; $500 \mathrm{ng} / \mathrm{mouse}$ ) a carotid artery catheter was placed for blood sampling (ProCyte Dx; IDEXX Laboratories). Thereafter, the cremaster muscle was exteriorized, and rolling flux fraction (rolling cells/ min divided by total neutrophil flux), rolling velocity, and leukocyte adhesion efficiency (number of adherent cells/mm divided by the systemic neutrophil count) were determined by intravital microscopy (BX51WI microscope, water immersion objective $\times 40,0.80 \mathrm{NA}$, Olympus; CCD camera, CF8/1, Kappa). Postcapillary venules ranged from 20 to $40 \mu \mathrm{m}$ in diameter.

Murine ex vivo flow chamber system and LFA-1 clustering. To investigate adhesion under flow conditions, we used a previously described flow chamber system (53). Glass capillaries (Rectangle Borosilicate Capillaries, $0.04 \times 0.40 \mathrm{~mm}$ inner diameter; VitroCom) were coated with E-selectin (R\&D Systems; $20 \mu \mathrm{g} / \mathrm{ml}$ ), ICAM-1 (R\&D Systems; 15 $\mu \mathrm{g} / \mathrm{ml}$ ), and/or CXCL1 (PeproTech; $15 \mu \mathrm{g} / \mathrm{ml}$ ). Flow chambers were autoperfused with whole blood from WT or $\mathrm{Mst1}^{-/-}$mice via a carotid artery catheter. One representative field of view was recorded by intravital microscopy (water immersion objective $\times 20,0.95$ NA; Olympus).

In order to investigate LFA-1 clustering during neutrophil adhesion under flow conditions, flow chambers were perfused $(2 \mu \mathrm{l} / \mathrm{min})$ with whole blood from WT or $\mathrm{Mst1}^{-/-}$mice, incubated with rat antiLFA-1 Alexa Fluor 546 antibody (2D7; BioLegend). Adherent cells were imaged using confocal microscopy (Leica SP5 system, $\times 63,1.4$ NA oil objective; Leica Microsystems).

Human flow chamber system. In order to investigate adhesion of human neutrophils under flow conditions, we used a previously described flow chamber system (54). Glass capillaries (Rectangle Borosilicate Capillaries, $0.2 \times 2 \mathrm{~mm}$ inner diameter; VitroCom) were coated with combinations of E-selectin (R\&D Systems; $5 \mu \mathrm{g} / \mathrm{ml}$ ), ICAM-1 (R\&D Systems; $4 \mu \mathrm{g} / \mathrm{ml}$ ), and CXCL8 (PeproTech; $5 \mu \mathrm{g} / \mathrm{ml}$ ). Flow chambers were perfused $\left(2 \mathrm{dyn} / \mathrm{cm}^{2}\right)$ with Ficoll purified neutrophils $\left(2 \times 10^{5} / \mathrm{ml}\right)$ from parents $\left(S T K 4^{+/-}\right)$or $S T K 4^{-/}$patients.

Neutrophil viability. Isolated neutrophils were cultured in HBSS buffer (containing $0.1 \%$ glucose, $1 \mathrm{mM} \mathrm{CaCl}_{2}$ and $\mathrm{MgCl}_{2}, 0.25 \%$ BSA [GE Healthcare], and $10 \mathrm{mM}$ HEPES [Sigma-Aldrich] $\mathrm{pH}$ 7.4) for 0,
24, 48, and 72 hours. Viability and apoptosis rate of Ly6G $\mathrm{G}^{+}$neutrophils were evaluated using TMRE (tetramethylrhodamine) and TO-PRO3 iodide (Invitrogen), respectively, and assessed by flow cytometry.

Viability of neutrophils within the cremaster muscle. To evaluate the viability of neutrophils within the whole cremaster muscle, TNF- $\alpha$ (500 ng/mouse) was injected into the scrotum of WT and $\mathrm{Mst}^{-/-}$mice. Two hours later, the cremaster muscle was exteriorized, dissected, and digested with Liberase $\left(0.25 \mathrm{mg} / \mathrm{ml}\right.$; Roche) for 1 hour at $37^{\circ} \mathrm{C}$. Neutrophils were stained using rat anti-Ly6G antibody and TO-PRO3 and analyzed using flow cytometry.

Surface expression of LFA-1, Mac-1, CXCR2, CD44, PSGL1, and CD62L. Surface expression of $\alpha_{\mathrm{L}}$ (LFA-1, CD11a-allophycocyanin, M17/4; eBioscience), $\alpha_{M}$ (Mac-1, CD11b-Brilliant Violet 570, M1/70; BioLegend), CXCR2 (CD182-allophycocyanin, 242216; R\&D Systems), CD44 (CD44-Brilliant Violet 570, IM7; BioLegend), PSGL1 (CD162-phycoerythrin, 2PH1; Pharmingen), and L-selectin (CD62LFITC, MEL-14; BioLegend) of bone marrow-derived Ly6G+ (Ly6GPacific Blue, 1A8; BioLegend) neutrophils from WT and $\mathrm{Mst1}^{-/-}$mice was compared using a Beckman Coulter Gallios flow cytometer and analyzed using Kaluza Flow Analysis Software (Beckman Coulter).

Soluble ICAM-1 binding assay. Soluble ICAM-1 binding assay was performed as described previously (52). Briefly, bone marrow-derived neutrophils were isolated from WT and $\mathrm{Mst}^{-/-}$mice using a Percoll gradient (Sigma-Aldrich). Cells were suspended in HBSS buffer. Cells were stimulated with $100 \mathrm{ng} / \mathrm{ml} \mathrm{rmCXCL1}$ (PeproTech) or an equal volume of HBSS buffer, in the presence of rmICAM-1 (ICAM-1 hFc chimera; R\&D Systems; $20 \mu \mathrm{g} / \mathrm{ml}$ ), goat anti-human Fc $\gamma$-biotin (polyclonal; eBioscience), and streptavidin-PerCP-Cy5.5 (eBioscience), for 3 minutes at $37^{\circ} \mathrm{C}$. Cells were fixed (FACS Lysing Solution; BD), stained with rat anti-mouse Ly6G-Pacific Blue antibody (1A8; BioLegend), and measured using a flow cytometer.

TNF- $\alpha$-induced neutrophil extravasation into the peritoneal cavity. WT and $\mathrm{Mst1}^{-/-}$mice were injected i.p. with $0.9 \% \mathrm{NaCl}$ (control) or TNF- $\alpha$ (rmTNF- $\alpha$; R\&D Systems; $2 \mu \mathrm{g} /$ mouse). Mice were sacrificed 2 hours later, and peritoneal lavage was performed, cells were collected, and the number of extravasated neutrophils was evaluated using rat anti-Ly6G antibody (1A8; BioLegend), Flow-Count Fluorospheres (Beckman Coulter), and flow cytometry. In a second set of experiments, WT mice were pretreated for 24 hours with the NE inhibitor GW311616A (BOC Sciences; $2 \mathrm{mg} / \mathrm{kg}$, orally) (55), followed by i.v. injection of the VLA-3-blocking peptide LXY2 (Bachem; H-cyclo[D-Cys-DAsp-Gly-Tyr(3-NO $\mathrm{N}_{2}$-Gly-ProOH-Asn-D-Cys]- $\mathrm{NH}_{2}, 88 \mu \mathrm{g}$ ) (16) and the $\mathrm{mAb}$ against $\alpha_{6}$ integrin (GoH3; BioLegend; $3 \mathrm{mg} / \mathrm{kg}$ ) (17) 15 minutes before i.p. administration of TNF- $\alpha$ ( $2 \mu \mathrm{g} /$ mouse). Control mice were pretreated for 24 hours with solvent, followed by i.v. injection of the control peptide (Bachem; H-Gly-Arg-Gly-Glu-Ser-OH, $88 \mu \mathrm{g}$ ) and isotype-matched control mAb (BioLegend; $3 \mathrm{mg} / \mathrm{kg}$ ) 15 minutes before i.p. administration of TNF- $\alpha$ ( $2 \mu \mathrm{g} / \mathrm{mouse})$. Two hours later, the number of extravasated neutrophils was evaluated using flow cytometry.

Multiphoton microscopy of the mouse cremaster muscle. Extravasation of neutrophils into inflamed mouse cremaster muscle of $L y z 2^{\text {GFP }}$ and $L y z 2^{G F P} \times M s t 1^{-/}$mice was investigated by multiphoton microscopy. Briefly, TNF- $\alpha$ (R\&D Systems; $500 \mathrm{ng} /$ mouse) and a rat antiPECAM-1 antibody Alexa Fluor 546 (390; BioLegend; $3 \mu \mathrm{g}$ ) were injected into the scrotum of mice. One hour later, the cremaster muscle was prepared for intravital multiphoton microscopy. Z-stacks were captured using a TrimScope (LaVision Bio Tec [ref. 56], upgraded with 
Hamamatsu H7422A-40 high-sensitivity GaAsP photomultipliers; Olympus XLUMPlanFI, $\times 20,0.95 \mathrm{~W}$ objective, $810 \mathrm{~nm}$ excitation, ultrasensitive port 525/50, 580/60). Images ( $966 \times 966$ pixels, 300 $\times 300 \mu \mathrm{m}$, step size $4 \mu \mathrm{m}$ ) were acquired at intervals of 30 seconds. After acquisition, images were processed and analyzed using Imaris 7 (Bitplane). Transmigration time was defined from the point when the cells started to transmigrate (excluding intraluminal crawling) until they completely detached from the abluminal vascular wall.

Laser-induced injury using multiphoton microscopy in the mouse cremaster muscle. A laser injury using high laser power by scanning of an area of $50 \times 50 \mu \mathrm{m} 121$ times in the center of $500 \times 500 \mu \mathrm{m}$ was set, 3 hours after intrascrotal injection of rmTNF- $\alpha$ and anti-PECAM-1-Alexa Fluor 546 antibody. Images were acquired for 30 minutes as described above. The GFP intensity, which is proportional to the number of neutrophils at the site of injury, was analyzed using Fiji software (57).

Chemotaxis assay for neutrophils in 3D collagen gels. 3D chemotaxis was performed according to the manufacturer's instructions (Ibidi). Briefly, bone marrow-derived neutrophils from WT and $\mathrm{Mst1}^{-/-}$mice were isolated using a Percoll gradient, and $3 \times 10^{5}$ cells were seeded together with collagen I into a channel of Ibidi $\mu$-Slides Chemotaxis ${ }^{3 \mathrm{D}}$. After 5 minutes of incubation at $37^{\circ} \mathrm{C}$, the reservoirs were filled with either complete HBSS buffer alone or rmCXCL1 (PeproTech; $100 \mathrm{ng} /$ $\mathrm{ml}$ ) or fMLP (Sigma-Aldrich; $10 \mu \mathrm{M}$ ). Images were acquired every 14 seconds for 30 minutes at $37^{\circ} \mathrm{C}$ using an Axiovert 200M microscope. Images were analyzed using Fiji software.

H. pylori infection model. WT or $\mathrm{Mst1}^{-/-}$mice were infected with the H. pylori strain PMSS1wt orogastrically 3 times with $10^{9}$ bacteria or sterile Brucella Broth (BD) alone as a control. Animals were sacrificed after 6 weeks or 3 months. Stomachs were opened, washed with PBS, and divided into 2 equal halves. One half was homogenized, and appropriate dilutions were spread on selective serum plates (Difco GC agar [BD] with horse serum [80 ml/l], IsoVitaleX [10 ml/1; BD, Germany], nalidixic acid [5 g/l], bacitracin [50 g/l], and Dent [Oxoid]). The number of CFU was calculated per gram of gastric tissue. The other half of the stomach was fixed in Tissue-Tek OCT Compound (Sakura), stained with a primary rat anti-Ly6G antibody (BD Pharmingen), and detected using a goat anti-rat-Alexa Fluor 555 antibody (Invitrogen) together with DAPI (Sigma-Aldrich). Images were obtained using an Olympus fluorescence microscope ( $\times 10,0.40$ NA dry objective). In a second set of experiments, the sections were stained with a rat antiPECAM-1-Alexa Fluor 488 antibody (clone 390; BioLegend) together with a rat anti-Ly6G antibody (BD Pharmingen) detected with a goat anti-rat-Alexa Fluor 555 antibody (Invitrogen). DAPI was used for nuclear staining. Sections were imaged by confocal microscopy (Leica SP5 system, $\times 40,1.25$ NA oil objective). The number of neutrophils was evaluated using Volocity software.

Analysis of neutrophil extravasation. TNF- $\alpha$ (R\&D Systems; $500 \mathrm{ng} /$ mouse) was applied to the scrotum of WT and $\mathrm{Mst1}^{-1}$ mice. After 2 hours the cremaster muscle was dissected and fixed with $4 \%$ paraformaldehyde for 1 hour, permeabilized and blocked for 2 hours in $0.5 \%$ Triton X-100/2\% ovalbumin/PBS, and incubated with rat anti-laminin $\alpha 5$ antibody $4 \mathrm{G} 6$ (58), rabbit anti-ESAM1 antibody (9), and goat anti-MRP14 antibody (S100A9; R\&D Systems) overnight in $2 \%$ ovalbumin/PBS at room temperature. After labeling with secondary antibodies (donkey anti-rat-Alexa Fluor 488, donkey anti-goat-Alexa Fluor 568, donkey anti-rabbit-Alexa Fluor 647; Invitrogen) in 1\% ovalbumin/PBS for 5 hours at room temperature, the tissue was embedded on glass slides in Dako Fluorescence Mounting Medium. Images were acquired using a confocal microscope (Zeiss LSM 780, LSM 510) and analyzed with Imaris software.

Transwell assay. HBSS buffer and $10 \mathrm{ng} / \mathrm{ml}$ or $100 \mathrm{ng} / \mathrm{ml}$ CXCL1 were used as chemoattractant in the Transwell system $(5-\mu \mathrm{m}$ pore size). Isolated bone marrow neutrophils (EasySep mouse neutrophil enrichment kit; STEMCELL Technologies) were applied and allowed to migrate for 45 minutes at $37^{\circ} \mathrm{C}$. Numbers of transmigrated neutrophils were evaluated using rat anti-Ly6G antibody (1A8; BioLegend), Flow-Count Fluorospheres, and flow cytometry. In a second set of experiments, Transwells ( $3-\mu \mathrm{m}$ pore size) were additionally coated with laminin 1 (Life Technologies; $15 \mu \mathrm{g} / \mathrm{ml}$ ) or 2\% BSA (GE Healthcare) as a control at $4^{\circ} \mathrm{C}$ overnight. Laminin was additionally coated with PECAM-1 (R\&D Systems; $2 \mu \mathrm{g} / \mathrm{ml}$ ) and ICAM-1 (R\&D Systems; $8 \mu \mathrm{g} / \mathrm{ml}$ ) for 2 hours at $37^{\circ} \mathrm{C}$. Using HBSS buffer alone (control) or HBSS buffer with $1 \mathrm{ng} / \mathrm{ml} \mathrm{CXCL1}$ as chemoattractant, isolated bone marrow neutrophils $\left(2 \times 10^{5}\right.$ cells per well) were allowed to migrate for 3 hours at $37^{\circ} \mathrm{C}(17)$. Numbers of transmigrated neutrophils were evaluated using rat anti-Ly6G antibody, Flow-Count Fluorospheres, and flow cytometry. In a third set of experiments, murine endothelial 1 G11 cells (provided by Annunciata Vecchi, Humanitas Research Hospital, Milan, Italy) were seeded on Transwell inserts (5- $\mu \mathrm{m}$ pore size). Either HBSS buffer alone or HBSS buffer containing $100 \mathrm{ng} / \mathrm{ml}$ CXCL1 was added to the lower compartments. After 30 minutes of equilibration, isolated bone marrow neutrophils $\left(2 \times 10^{5}\right.$ cells per well $)$ were allowed to migrate for 1 hour at $37^{\circ} \mathrm{C}$. Numbers of transmigrated cells were evaluated using rat anti-Ly6G antibody and Flow-Count Fluorospheres with flow cytometry.

Mobilization of VLA-3, VLA-6, NE, MST1, and Rab27a in mouse neutrophils. Mobilization of NE, $\alpha_{3}$ (VLA-3, also known as CD49c), and $\alpha_{6}$ (VLA-6, also known as CD49f) on PECAM-1-, ICAM-1-, and CXCL1-coated wells was performed as previously described (17). Briefly, slides were coated with either $2 \%$ BSA (control; GE Healthcare) or PECAM-1 (2 $\mu \mathrm{g} / \mathrm{ml}$; R\&D Systems), ICAM-1 (8 $\mu \mathrm{g} / \mathrm{ml}$; R\&D Systems), and CXCL1 (10 $\mu \mathrm{g} / \mathrm{ml}$; PeproTech) overnight at $4^{\circ} \mathrm{C}$. Isolated bone marrow neutrophils from WT, $\mathrm{Mst1}^{-/}, \mathrm{Rab} 27 \mathrm{a}^{\text {ash } / a s h}$, Sytl1 ${ }^{-/}$, and Munc13-4inx/jinx mice were incubated on coated slides for 30 minutes at $37^{\circ} \mathrm{C}$. Cells were fixed with $4 \%$ paraformaldehyde, blocked and permeabilized with PBS/0.1\% Triton X-100/2\% BSA, and stained with a mouse anti-VLA-3 antibody (42/CD49c; BD), rat anti-VLA-6 antibody (GoH3; BioLegend), rabbit anti-NE antibody (polyclonal; Abcam), sheep anti-Rab27a antibody (R\&D Systems), and rabbit anti-MST1 antibody (polyclonal; Cell Signaling Technology). Primary antibodies were detected using goat anti-mouse-Alexa Fluor 488, goat anti-rabbit-Alexa Fluor 546, donkey anti-sheep-Alexa Fluor 488, and goat anti-rat-Alexa Fluor 647 (Molecular Probes/Invitrogen), respectively. Cells were embedded in PermaFluor (Thermo Scientific) and imaged by confocal microscopy (Leica SP5 System, $\times 63,1.4$ NA oil objective). To investigate the translocation of VLA-3, VLA-6, NE, MST1, and Rab27a, fluorescence intensity profiles were obtained along a line through the cell as exemplified in Supplemental Figure 3A.

In vivo NE activity assay. NE680FAST (4 nmol/mouse; PerkinElmer) was injected i.v. TNF- $\alpha$ (rmTNF- $\alpha$; R\&D Systems; 500 ng/ mouse) was applied to the scrotum of WT and $\mathrm{Mst1}^{-/-}$mice 1 hour later. Two hours after TNF- $\alpha$ injection, the cremaster muscle was dissected and fixed with $4 \%$ paraformaldehyde, permeabilized and blocked 
with $0.5 \%$ Triton $\mathrm{X}-100 / 2 \%$ ovalbumin/PBS, stained with rat antiPECAM-1 antibody-Alexa Fluor 488 (MEC13.3; BioLegend) or rabbit anti-MRP14 antibody (gift of Thomas Vogl, University of Münster, Münster, Germany), and detected with a goat anti-rabbit-Alexa Fluor 546 antibody (Invitrogen). Tissue was embedded on glass slides in PermaFluor (Thermo Scientific) and imaged by confocal microscopy (Leica SP5 system, $\times 40,1.4$ NA oil objective).

Neutrophil fractionation. Human blood neutrophils were isolated by Ficoll density centrifugation as previously described (59). For sucrose density gradient fractionation, supernatants of neutrophil lysates were placed on top of a continuous sucrose gradient $(20 \%$ $70 \%$ ) and spun down at $150,000 \mathrm{~g}$ for 1 hour at $4^{\circ} \mathrm{C}$. Aliquots were collected from top to bottom and analyzed for the expression of MST1, Rab27a, and myeloperoxidase (24).

Statistics. All data were analyzed and plotted using GraphPad Prism 6 software (GraphPad Software Inc.). For pairwise comparison of experimental groups, a paired $t$ test, unpaired $t$ test, or Mann-Whitney test was performed. For comparison of 3 or more samples, a 1-way ANOVA was used with Dunnett's multiple comparisons test. For multiple comparisons, a 2-way ANOVA was used with either Sidak's multiple comparisons test or Tukey's multiple comparisons test (comparison of all experimental groups against each other). A $P$ value less than 0.05 was considered as statistically significant.

Study approval. $\mathrm{Mst1}^{-/-}$mice were generated by Dae-Sik Lim as described (60). Lyz $2^{\text {GFP }}$ mice (36) were generously provided by Thomas Graf (Centre for Genomic Regulation, Barcelona, Spain). Lyz $2^{\text {GFP }} \times$ $M s t 1^{-/-}$mice were generated by crossbreeding. Rab27 $a^{\text {ash } / a s h}$ (61), Sytl1 ${ }^{-1-}$ (50), and Munc13-4 jinx/jinx $(22,62)$ knockout mice were housed by Sergio D. Catz (The Scripps Research Institute). Studies with these mice were conducted according to NIH and institutional guidelines and with approval from the animal review board at The Scripps Research
Institute. C57BL/6 WT mice were obtained from Janvier Labs. Animal studies were approved by the Regierung von Oberbayern, Germany (AZ 55.2-1-54-2531-149/10 and AZ 55.2-1-54-2532-34/14).

Human studies with purified neutrophils from parents $\left(S T K 4^{+-}\right)$, $S T K 4^{--}$patients, and adult healthy volunteers were approved by the ethics committee of Ludwig Maximilian University (AZ 66-14 and AZ 611-15).

\section{Author contributions}

ARMK, MP, and IR designed, performed, and analyzed experiments, interpreted data, and wrote the manuscript; MR, KS, UH, GG, and CN performed and analyzed experiments; BW, RI, JRW, and AM analyzed experiments; DSL and SD provided reagents critical for the project; $\mathrm{MM}, \mathrm{CK}, \mathrm{DV}$, and $\mathrm{RH}$ contributed to the design of the experiments and the interpretation of the data; and MS and SDC designed experiments, interpreted data, and wrote the manuscript.

\section{Acknowledgments}

We thank Susanne Bierschenk, Nadine Schmidt, Eva Loell, and Jennifer Truong for excellent technical assistance. This work was supported by DFG SP621/5-1 (to MS); SFB914, projects A01 (MM), A02 (BW), A08 (CK), B01 (MS), and B05 (RH); the Care-for-Rare Foundation (CK); the EU Project Tarkinaid FP7-Health.2011.1.4.5 \#282095 (MS, BW); US Public Health Service grants HL088256 and GM105894 (to SDC); and an American Heart Association fellowship (to MR).

Address correspondence to: Markus Sperandio, Walter Brendel Center of Experimental Medicine, Ludwig-Maximilians-Universität, Marchioninistr. 15, 81377 Munich, Germany. Phone: 49.0.89.2180.76505; E-mail: markus.sperandio@lmu.de.
1. Nourshargh S, Alon R. Leukocyte migration into inflamed tissues. Immunity. 2014;41(5):694-707.

2. Kolaczkowska E, Kubes P. Neutrophil recruitment and function in health and inflammation. Nat Rev Immunol. 2013;13(3):159-175.

3. Hallmann R, et al. The regulation of immune cell trafficking by the extracellular matrix. Curr Opin Cell Biol. 2015;36:54-61.

4. Rowe RG, Weiss SJ. Breaching the basement membrane: who, when and how? Trends Cell Biol. 2008;18(11):560-574.

5. Sorokin L. The impact of the extracellular matrix on inflammation. Nat Rev Immunol. 2010;10(10):712-723.

6. Muller WA. Localized signals that regulate transendothelial migration. Curr Opin Immunol. 2016;38:24-29.

7. Bixel MG, et al. CD99 and CD99L2 act at the same site as, but independently of, PECAM-1 during leukocyte diapedesis. Blood. 2010;116(7):1172-1184.

8. Thompson RD, et al. Platelet-endothelial cell adhesion molecule-1 (PECAM-1)-deficient mice demonstrate a transient and cytokine-specific role for PECAM-1 in leukocyte migration through the perivascular basement membrane. Blood. 2001;97(6):1854-1860.

9. Wegmann F, et al. ESAM supports neutrophil extravasation, activation of Rho, and VEGF- induced vascular permeability. J Exp Med. 2006;203(7):1671-1677.

10. Sixt M, Engelhardt B, Pausch F, Hallmann R, Wendler O, Sorokin LM. Endothelial cell laminin isoforms, laminins 8 and 10, play decisive roles in $\mathrm{T}$ cell recruitment across the blood-brain barrier in experimental autoimmune encephalomyelitis. JCell Biol. 2001;153(5):933-946.

11. Wu C, et al. Endothelial basement membrane laminin alpha5 selectively inhibits T lymphocyte extravasation into the brain. Nat Med. 2009;15(5):519-527.

12. Kenne E, Soehnlein O, Genové G, Rotzius P, Eriksson EE, Lindbom L. Immune cell recruitment to inflammatory loci is impaired in mice deficient in basement membrane protein laminin alpha4. J Leukoc Biol. 2010;88(3):523-528.

13. Wang $\mathrm{S}$, et al. Venular basement membranes contain specific matrix protein low expression regions that act as exit points for emigrating neutrophils. JExp Med. 2006;203(6):1519-1532.

14. Hyun YM, et al. Uropod elongation is a common final step in leukocyte extravasation through inflamed vessels. J Exp Med. 2012;209(7):1349-1362.

15. Dangerfield JP, Wang S, Nourshargh S. Blockade of alpha6 integrin inhibits IL-1beta- but not TNF-alpha-induced neutrophil transmigration in vivo. JLeukoc Biol. 2005;77(2):159-165.
16. Lerman YV, et al. Sepsis lethality via exacerbated tissue infiltration and TLR-induced cytokine production by neutrophils is integrin $\alpha 3 \beta 1$ dependent. Blood. 2014;124(24):3515-3523.

17. Wang S, Dangerfield JP, Young RE, Nourshargh S. PECAM-1, alpha6 integrins and neutrophil elastase cooperate in mediating neutrophil transmigration. J Cell Sci. 2005;118(Pt 9):2067-2076.

18. Young RE, Voisin MB, Wang S, Dangerfield J, Nourshargh S. Role of neutrophil elastase in LTB4-induced neutrophil transmigration in vivo assessed with a specific inhibitor and neutrophil elastase deficient mice. Br J Pharmacol. 2007;151(5):628-637.

19. Uriarte SM, et al. Comparison of proteins expressed on secretory vesicle membranes and plasma membranes of human neutrophils. JImmunol. 2008;180(8):5575-5581.

20. Catz SD. Regulation of vesicular trafficking and leukocyte function by Rab27 GTPases and their effectors. J Leukoc Biol. 2013;94(4):613-622.

21. Catz SD. The role of Rab27a in the regulation of neutrophil function. Cell Microbiol. 2014;16(9):1301-1310.

22. Brzezinska AA, et al. The Rab27a effectors JFC1/ Slp1 and Munc13-4 regulate exocytosis of neutrophil granules. Traffic. 2008;9(12):2151-2164.

23. Johnson JL, Hong H, Monfregola J, Kiosses WB, Catz SD. Munc13-4 restricts motility of 
Rab27a-expressing vesicles to facilitate lipopolysaccharide-induced priming of exocytosis in neutrophils. J Biol Chem. 2011;286(7):5647-5656.

24. Munafó DB, Johnson JL, Ellis BA, Rutschmann S, Beutler B, Catz SD. Rab27a is a key component of the secretory machinery of azurophilic granules in granulocytes. Biochem J. 2007;402(2):229-239.

25. Pivot-Pajot C, Varoqueaux F, de Saint Basile G, Bourgoin SG. Munc13-4 regulates granule secretion in human neutrophils. JImmunol. 2008;180(10):6786-6797.

26. Abdollahpour H, et al. The phenotype of human STK4 deficiency. Blood. 2012;119(15):3450-3457.

27. Crequer A, et al. Inherited MST1 deficiency underlies susceptibility to EV-HPV infections. PLOS ONE. 2012;7(8):e44010.

28. Nehme NT, et al. MST1 mutations in autosomal recessive primary immunodeficiency characterized by defective naive T-cell survival. Blood. 2012;119(15):3458-3468.

29. Nishikimi A, et al. Rab13 acts downstream of the kinase Mst1 to deliver the integrin LFA-1 to the cell surface for lymphocyte trafficking. Sci Signal. 2014;7(336):ra72.

30. Katagiri K, Imamura M, Kinashi T. Spatiotemporal regulation of the kinase Mst1 by binding protein RAPL is critical for lymphocyte polarity and adhesion. Nat Immunol. 2006;7(9):919-928.

31. Katagiri K, Katakai T, Ebisuno Y, Ueda Y, Okada T, Kinashi T. Mst1 controls lymphocyte trafficking and interstitial motility within lymph nodes. ЕМВО J. 2009;28(9):1319-1331.

32. De Souza PM, Kankaanranta H, Michael A, Barnes PJ, Giembycz MA, Lindsay MA. Caspase-catalyzed cleavage and activation of Mst1 correlates with eosinophil but not neutrophil apoptosis. Blood. 2002;99(9):3432-3438.

33. Choi J, et al. Mst1-FoxO signaling protects Naïve $\mathrm{T}$ lymphocytes from cellular oxidative stress in mice. PLoS ONE. 2009;4(11):e8011.

34. Dong Y, et al. A cell-intrinsic role for Mst1 in regulating thymocyte egress. J Immunol. 2009;183(6):3865-3872.

35. Woodfin A, et al. The junctional adhesion molecule JAM-C regulates polarized transendothelial migration of neutrophils in vivo. Nat Immunol. 2011;12(8):761-769.

36. Faust N, Varas F, Kelly LM, Heck S, Graf T. Insertion of enhanced green fluorescent protein into the lysozyme gene creates mice with green fluorescent granulocytes and macrophages. Blood. 2000;96(2):719-726.

37. Lämmermann T, et al. Neutrophil swarms require
LTB4 and integrins at sites of cell death in vivo. Nature. 2013;498(7454):371-375.

38. D'Elios MM, Amedei A, Cappon A, Del Prete G, de Bernard $M$. The neutrophil-activating protein of Helicobacter pylori (HP-NAP) as an immune modulating agent. FEMS Immunol Med Microbiol. 2007;50(2):157-164.

39. Montecucco C, Rappuoli R. Living dangerously: how Helicobacter pylori survives in the human stomach. Nat Rev Mol Cell Biol. 2001;2(6):457-466.

40. Suerbaum S, Michetti P. Helicobacter pylori infection. N Engl JMed. 2002;347(15):1175-1186.

41. Yousif LF, Di Russo J, Sorokin L. Laminin isoforms in endothelial and perivascular basement membranes. Cell Adh Migr. 2013;7(1):101-110.

42. Sheshachalam A, Srivastava N, Mitchell T, Lacy P, Eitzen G. Granule protein processing and regulated secretion in neutrophils. Front Immunol. 2014;5:448.

43. Yu FX, Zhao B, Guan KL. Hippo pathway in organ size control, tissue homeostasis, and cancer. Cell. 2015;163(4):811-828.

44. Fabbri M, et al. Dynamic partitioning into lipid rafts controls the endo-exocytic cycle of the alphaL/beta2 integrin, LFA-1, during leukocyte chemotaxis. Mol Biol Cell. 2005;16(12):5793-5803.

45. Dangerfield J, Larbi KY, Huang MT, Dewar A, Nourshargh S. PECAM-1 (CD31) homophilic interaction up-regulates alpha6beta1 on transmigrated neutrophils in vivo and plays a functional role in the ability of alpha6 integrins to mediate leukocyte migration through the perivascular basement membrane. JExp Med. 2002;196(9):1201-1211.

46. Johnson JL, et al. Rab27a and Rab27b regulate neutrophil azurophilic granule exocytosis and NADPH oxidase activity by independent mechanisms. Traffic. 2010;11(4):533-547.

47. Barral DC, et al. Functional redundancy of Rab27 proteins and the pathogenesis of Griscelli syndrome. JClin Invest. 2002;110(2):247-257.

48. Saegusa C, Kanno E, Itohara S, Fukuda M. Expression of Rab27B-binding protein Slp1 in pancreatic acinar cells and its involvement in amylase secretion. Arch Biochem Biophys. 2008;475(1):87-92.

49. Shirakawa R, et al. Munc13-4 is a GTPRab27-binding protein regulating dense core granule secretion in platelets. J Biol Chem. 2004;279(11):10730-10737.

50. Johnson JL, Monfregola J, Napolitano G, Kiosses WB, Catz SD. Vesicular trafficking through cortical actin during exocytosis is regulated by the Rab27a effector JFC1/Slp1 and the RhoAGTPase-activating protein Gem-interacting protein. Mol Biol Cell. 2012;23(10):1902-1916.

51. Johnson JL, Hong H, Monfregola J, Kiosses WB, Catz SD. Munc13-4 restricts motility of Rab27a-expressing vesicles to facilitate lipopolysaccharide-induced priming of exocytosis in neutrophils. J Biol Chem. 2011;286(7):5647-5656.

52. Pruenster M, et al. Extracellular MRP8/14 is a regulator of $\beta 2$ integrin-dependent neutrophil slow rolling and adhesion. Nat Commun. 2015;6:6915.

53. Yang WH, Nussbaum C, Grewal PK, Marth JD, Sperandio M. Coordinated roles of ST3Gal-VI and ST3Gal-IV sialyltransferases in the synthesis of selectin ligands. Blood. 2012;120(5):1015-1026.

54. Nussbaum C, et al. Neutrophil and endothelial adhesive function during human fetal ontogeny. JLeukoc Biol. 2013;93(2):175-184.

55. Colom B, et al. Leukotriene B4-Neutrophil Elastase Axis Drives Neutrophil Reverse Transendothelial Cell Migration In Vivo. Immunity. 2015;42(6):1075-1086.

56. Rehberg M, Krombach F, Pohl U, Dietzel S. Label-free 3D visualization of cellular and tissue structures in intact muscle with second and third harmonic generation microscopy. PLOS ONE. 2011;6(11):e28237.

57. Schindelin J, et al. Fiji: an open-source platform for biological-image analysis. Nat Methods. 2012;9(7):676-682.

58. Sorokin LM, Pausch F, Frieser M, Kröger S, Ohage E, Deutzmann R. Developmental regulation of the laminin alpha5 chain suggests a role in epithelial and endothelial cell maturation. Dev Biol. 1997;189(2):285-300.

59. Markert M, Andrews PC, Babior BM. Measurement of $\mathrm{O} 2$ - production by human neutrophils. The preparation and assay of NADPH oxidase-containing particles from human neutrophils. Meth Enzymol. 1984;105:358-365.

60. Oh S, et al. Crucial role for Mst1 and Mst2 kinases in early embryonic development of the mouse. Mol Cell Biol. 2009;29(23):6309-6320.

61. Johnson JL, et al. Rab27a and Rab27b regulate neutrophil azurophilic granule exocytosis and NADPH oxidase activity by independent mechanisms. Traffic. 2010;11(4):533-547.

62. Crozat K, et al. Jinx, an MCMV susceptibility phenotype caused by disruption of Unc13d: a mouse model of type 3 familial hemophagocytic lymphohistiocytosis. JExp Med. 2007;204(4):853-863. 